\title{
Review
}

\section{Rupture, waves and earthquakes}

\author{
By Koji UenISHI ${ }^{* 1, \dagger}$
}

(Communicated by Yoshio FukaO, M.J.A.)

\begin{abstract}
Normally, an earthquake is considered as a phenomenon of wave energy radiation by rupture (fracture) of solid Earth. However, the physics of dynamic process around seismic sources, which may play a crucial role in the occurrence of earthquakes and generation of strong waves, has not been fully understood yet. Instead, much of former investigation in seismology evaluated earthquake characteristics in terms of kinematics that does not directly treat such dynamic aspects and usually excludes the influence of high-frequency wave components over $1 \mathrm{~Hz}$. There are countless valuable research outcomes obtained through this kinematics-based approach, but "extraordinary" phenomena that are difficult to be explained by this conventional description have been found, for instance, on the occasion of the 1995 Hyogo-ken Nanbu, Japan, earthquake, and more detailed study on rupture and wave dynamics, namely, possible mechanical characteristics of (1) rupture development around seismic sources, (2) earthquake-induced structural failures and (3) wave interaction that connects rupture (1) and failures (2), would be indispensable.
\end{abstract}

Keywords: earthquake, structural failure, wave interaction, mechanical stability, rupture speed

\section{Introduction}

Historically, utilizing the concepts of "continuum mechanics," the study in seismology has been developed to find more reasonable relations between (earth-)"quakes" (literally, "shaking" on the earth or "vibrations" in our living environments) and "waves" originating from seismic sources and propagating underground (and sometimes in the air). ${ }^{1)}$ However, like other dynamic fracture (rupture) phenomena in solid materials, an earthquake, i.e., rupture of solid Earth, most often happens at a "hidden" place and even when dynamic rupture can be observed, its fast development cannot be traced with naked eyes or normal video recording devices. Furthermore, multi-scale complexity of dynamic rupture makes it difficult to understand the essential mechanism of its development and related events. Therefore, in the theoretical investigation of conventional seismology, the physical process occurring around rupturing

\footnotetext{
*1 School of Engineering, The University of Tokyo, Tokyo, Japan.

$\dagger$ Correspondence should be addressed: K. Uenishi, School of Engineering, The University of Tokyo, 7-3-1 Hongo, Bunkyo-ku, Tokyo 113-8656, Japan (e-mail: uenishi@dyn.t.u-tokyo.ac.jp).
}

seismic sources is not directly treated and primary emphases are placed on the estimation of kinematic source parameters from seismological records, namely, seismograms. Even in the case rupture dynamics of sources is considered, the analyses are based on the results of the laboratory observations of one-dimensional frictional tests of sliding block surfaces, whose relation with real three-dimensional dynamic rupture in solid materials is actually still unknown. $\left.{ }^{2}\right)$ Moreover, especially in engineering applications, the difference between the terms "vibration" (or "oscillation") and "wave" is not clearly recognized, and in most structural analyses, seismological data, recorded by seismographs at nearby stations but low-pass filtered, are given straightforwardly to a target structure as vibration, and wave propagation in the structure, interaction between the structure and waves in the ground (and air) as well as their influence back to the seismograms is rarely handled. Since the theories and methods developed in this traditional, long-period or low-pass filtered seismology have been instructively and mathematically described in a textbook, ${ }^{3)}$ they are widely used in earthquake study, but it is difficult to apply this low-pass filtered seismology to the 
investigation of high-frequency waves over $1 \mathrm{~Hz}$ even when the quality, amount and computational processing abilities of seismological data are improved. ${ }^{4), 55}$

In order to overcome this difficulty, in the more recently developed stochastic approach, small-scale seismic inhomogeneities in the lithosphere are inferred and accepted from the existence of coda waves in the seismological data set. This method to handle the scattering of short-period or high-frequency seismic waves at small-scale inhomogeneities is summarized in order in another textbook, ${ }^{6)}$ and the existence of high-frequency seismic waves, those over $1 \mathrm{~Hz}$, is well recognized in earthquake science. On the other hand, the works in engineering seismology still remain traditional analyses of mechanical behavior of structures due to low-frequency horizontal "vibration." Even if the term "dynamic" is employed, in rock mechanics, for example, that term denotes quasi-static fatigue development under cyclic loading, where the effect of inertia is almost neglected. ${ }^{7)}$

Here, therefore, research works on earthquakerelated phenomena that are based on more rigorous rupture and wave dynamics are reviewed. The topics include mechanical destabilization of slowly developing seismic rupture sources, rupture propagation and wave radiation, and effects of waves on near-surface or surface exposed structures, or reversely, influence of such inhomogeneous structures on waves. The important role of higher-frequency seismic waves in generating structural failures at surface and in the underground, which has been nearly neglected in the engineering field, will be emphasized by introducing unique structural failure patterns "unexpectedly" found after several relatively large earthquakes in the world. This review summarizes rather nonconventional ideas about previously unrecognized, possible significance of rupture stability, fast rupture propagation and higher-frequency seismic waves in seismological study, but the objective is not to negate other valuable research results obtained through the standard methods.

\section{Seismic source at depth: universal mechanical instability for earthquake nucleation}

Although there are a diverse variety of earthquakes, fast and slow at various scales, ${ }^{8), 9)}$ seismological observations made possible by the worldwide installation of a standardized seismic network have supported the idea that majority of shallow earthquakes are caused by mechanical destabilization of geological faults. ${ }^{10)}$ The classical approach to understand earthquake rupture mechanisms started by assuming the preexistence of a single fault in a solid medium. Generally, the fault is assumed to be planar, and it is presumed that rupture on the fault plane (plane of weakness or interface) is controlled by friction. ${ }^{11)}$ In the earthquake nucleation process, a slowly (quasi-statically) expanding rupture region at a seismic source on a fault plane suddenly loses its mechanical stability and radiates seismic energy to the far field. A typical constitutive relation that holds inside the rupture region is the slip-weakening friction law where the stress inside the rupture region drops as slip (displacement discontinuity) develops on the fault plane. Another often employed friction law assumes an established fault surface and is described by a laboratory-derived rate- and statedependent friction law typically with slowness (or ageing) state evolution, ${ }^{12}$ ) and analytically as well as numerically, models based on slip-weakening or rate- and state-dependent constitutive relations have been proposed to study the earthquake initiation process, and in particular, the transition from stable quasi-static enlargement of the rupture region to unstable fast propagation of rupture. ${ }^{13)-17)}$ Numerically, this kind of problem has had intricacies, since the conventional quasi-static methods used for simulating slow and long-lasting deformation phases fail as instabilities develop. Hence, instead of handling the quasi-static state, the initiation of dynamic anti-plane slip instabilities of a slipweakening fault has been investigated by means of a spectral analysis for a homogeneous linear elastic medium that is preloaded uniformly up to the frictional threshold, and the effect of slip-weakening on the duration of the nucleation phase and the critical fault length has been evaluated. ${ }^{18), 19)}$ The objectives have been widened to in-plane shear instabilities $^{20)}$ and further to instabilities of a rupture region of (a priori) fixed length. ${ }^{21)}$ However, no statement concerning the physical meaning of the (a priori) fixed length and uniform preloading has been provided in these analyses, and rigorous numerical simulations of the whole earthquake cycle $^{22), 23)}$ have illustrated quasi-static expansion of a region of originally aseismic slip before dynamic rupture breakout. ${ }^{24)}$

Universal nucleation length associated with slip-weakening fault instabilities. For a more quantitative description of the difficulty in incorporating mechanical destabilization and initiation of dynamic stage of fault rupture in dynamicsbased seismological investigations, therefore, the factors that govern the nucleation of slip-weakening 


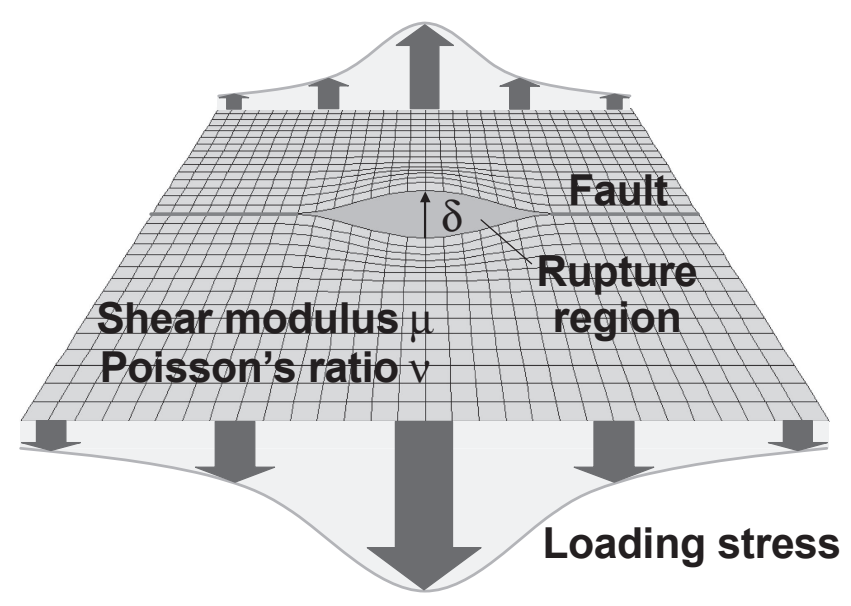

$\mathrm{b}$

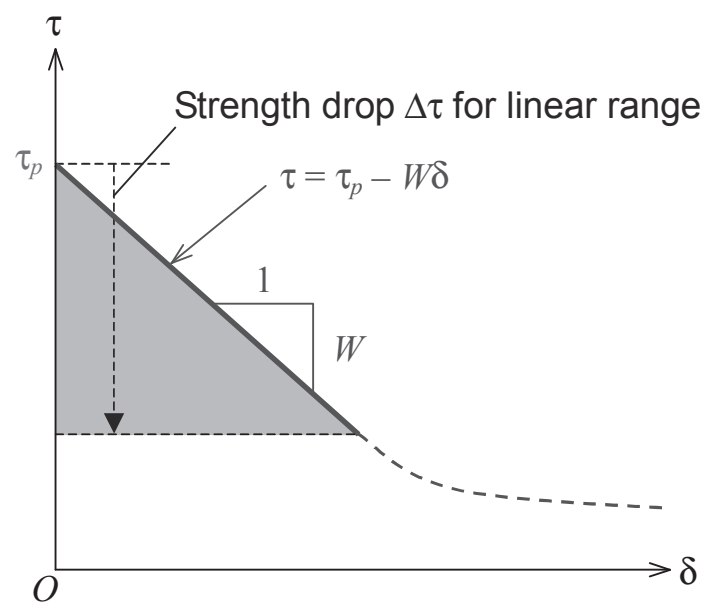

Fig. 1. (a) An analytical model for the nucleation of an earthquake on a geological fault. Contrary to our intuition, earthquakes owing to linear slip-weakening (see (b)) instabilities may spontaneously occur regardless of the precise distribution of the loading stress acting on the fault. Here, a displacement field is depicted for anti-plane shear (mode III) rupture in an infinite, homogeneous linear elastic medium. Similarly, the problem for in-plane shear (mode II) or tensile (mode I) rupture can be defined. (b) The (initially) linear slipweakening constitutive relation often employed in seismological investigations. The stress $\tau$ inside the rupture region of the fault obeys the linear relation $\tau=\tau_{p}-W \delta$, at least when the strength drop is less than $\Delta \tau$. Here, $\delta$ is slip (displacement discontinuity) on the fault plane, $W$ is constant slip-weakening rate $(W>0)$, and $\tau_{p}$ is the peak strength, i.e., slip can occur if the local shear stress $\tau$ reaches the peak shear strength for the shear modes II and III, and in a mode I tensile fracture interpretation, fracture opening can occur if tensile stress $\tau$ reaches $\tau_{p}{ }^{24)}$

instabilities have been identified for a two-dimensional planar fault (Fig. 1a). ${ }^{24)}$ The fault is under a loading stress that is locally peaked in space and increasing gradually with time, e.g., owing to tectonic loading, and the nucleation length that is relevant to fault instabilities and seismic rupture has been estimated. The size of the rupture region on the fault in this linear elastic model stably grows quasistatically under increasing load until it reaches a critical nucleation length at which no further solution for quasi-static elastic equilibrium exists. This corresponds to the start of a dynamically controlled instability. For the case of a widely used linear slip-weakening law (Fig. 1b), with $W$ being constant weakening rate $(W>0)$, the problem of slip-weakening fault instabilities has been reduced to an eigenvalue problem and the critical nucleation length $h_{n}$ relevant to the instabilities, i.e., earthquake nucleation, has been analytically proven to be universal. It is dependent on an elastic parameter of the medium and the slip-weakening rate $W$ only, and it is not affected by the strength of the fault plane, rate of increase of loading stress, or by the exact shape of the loading stress distribution along the fault. The nucleation length is given $b^{24)}$

$$
h_{n} \approx 1.158 \mu^{*} / W
$$

Here, $\mu^{*}=\mu$ (shear modulus) for mode III (antiplane shear remote loading; Fig. 1a), and $\mu /(1-\nu)$ for modes I (tensile loading) and II (in-plane shear loading), with $\nu$ being Poisson's ratio. Similar expressions can be found also for the three-dimensional case. ${ }^{25)}$

The difficulties in dynamically simulating earthquakes. In the formula for the universal nucleation length, Eq. [1], the slip-weakening rate $W$ plays an important role. This parameter is expressed as $W=\Delta \tau / D_{c}$ if linear weakening applies for slips $\delta<D_{c}$ and no further weakening exists for $\delta>D_{c}$, with the slip-weakening distance $D_{c}$ and the total strength drop $\Delta \tau$ (see Fig. $1 \mathrm{~b}$ ). While small values up to $1 \mathrm{~mm}$ are suggested for $D_{c}$ from laboratory testing of shear rupture of initially intact Fichtelbirge granite specimens ${ }^{26)-28)}$ and the associated nucleation length is estimated to be up to $h_{n} \approx 1 \mathrm{~m}$, relatively large values of $D_{c}$ (of order of one meter) are commonly inferred in kinematic seismic inversions. Equation [1] suggests that if a relatively large value of $D_{c}$, for instance, $D_{c}=0.05$ to $1 \mathrm{~m}$, is presumed, together with $\mu^{*}=40 \mathrm{GPa}$ and low strength drop $\Delta \tau=5$ to $15 \mathrm{MPa}$ that are archetypally employed in numerical simulations, ${ }^{29)-32}$ the related nucleation length is already as large as $h_{n} \approx 0.15$ to $9 \mathrm{~km}$. This size seems irreconcilable with presence of small- 
magnitude earthquakes. The result implies that the slip-weakening process may occur at slips much larger than those at initiation and the model with a single slip-weakening process may be insufficient. For example, it can be assumed that a laboratory-like weakening at small slips with a larger value of $W$ may govern nucleation, and then further weakening with smaller $W$ (e.g., owing to shear heating) may occur at much larger slips, ${ }^{33)}$ and this smaller $W$ is "detected" by the traditional seismic inversions. ${ }^{24}$ )

However, even when the linear slip-weakening law may be assumed, there are reasons why such often inferred large values of $D_{c}$ must be cautiously handled. First, from the observational viewpoint, it has been shown that assessments of $D_{c}$ inferred from kinematic seismic inversions tend to be biased towards large due to the influence of spatiotemporal smoothing constraints. ${ }^{34)}$ High frequencies, contaminated by scattering, are filtered out of the seismological records. It has been also indicated that $D_{c}$ is not uniquely determined by seismic inversions, ${ }^{34)}$ and so as to constrain $D_{c}$, ground motion spectra at frequencies higher than those currently normally possible must be modeled. Second, from the forward modeling viewpoint, $D_{c}$ is likely to be large owing to the technical problem in simulating earthquakes. A fault region, at least tens to hundreds of kilometers, must be simulated while slip as well as rapid stress change at the tips of propagating ruptures is being rightly resolved. ${ }^{22)}$ For instance, for a laboratoryderived value $D_{c}=800 \mu \mathrm{m}$, the dimension of fast slip buildup zone at the rupture front is some $1 \mathrm{~m},{ }^{27), 28}$ ) which requires 1000 times as many grid points as the calculation with $D_{c}=0.8 \mathrm{~m}$. Thus larger values of $D_{c}$ are likely to be used in regular numerical simulations. This implies that if forward dynamic simulations of earthquakes with computationally feasible grid size are coordinated to seismological data, a bias may arise towards large $D_{c} / \Delta \tau$ or small $W$ that can be resolved with such grid size, since numerical artifacts will contaminate any computations with smaller but possibly more realistic $D_{c} / \Delta \tau$ or larger $W^{24)}$ In these senses, it is difficult to perform really dynamic earthquake rupture simulations.

\section{Extraordinary fast rupture propagation and generation of high-frequency seismic waves}

Discrepancy between theoretical, experimental and seismologically inferred rupture propagation speeds. Once a rupture region loses its mechanical stability, its size extends dynamically. According to classical fracture mechanics, the theo- retical upper limit of the propagation speed $V$ of the tip of (continuously accelerating) planar rupture in a monolithic, homogenous linear elastic solid material is the Rayleigh surface wave speed $V_{R}$ (about $90 \%$ of the shear $(\mathrm{S})$ wave speed $V_{S}$, i.e., Mach number $V / V_{S} \approx 0.9$ ) of the material under typical mode I or II remote loading conditions. For mode III loading, the limit is slightly higher, $V_{S}$, and some kinematic seismic inversions even suggest the existence of supershear rupture speeds where rupture moves faster than the relevant $\mathrm{S}$ wave $\left(V / V_{S}>1\right) .{ }^{31), 35)-38)}$ However, the rupture speeds obtained by fracture experiments of monolithic brittle linear elastic solid materials like intact rocks are usually by far lower than those predicted by theories and inferred from inversions of seismograms. Laboratory experimental observations indicate that when rupture extends in brittle solid materials under suitable stress conditions and its propagation speed exceeds a certain value, it oscillates (rupture surface roughening) and afterwards divides into two or more branches. For tensile rupture in brittle amorphous solids (e.g., glass, polymethyl methacrylate (PMMA)), the rupture propagation speed $V$ has experimentally an upper limit of about $V / V_{S} \approx 0.5-0.6$. The rupture surface is mirror-smooth only for $V / V_{S}<0.27-0.36$. The rupture surface roughens severely at higher speeds and the rupture bifurcates at the highest speeds. ${ }^{39)-45)}$ Thus, it seems almost impossible to reach the "extraordinary" high theoretical limit speed in laboratory experiments, but there are at least two ways to increase the rupture propagation speed over the classical limit: One is to introduce precut interfaces in linear elastic materials and another is to consider nonlinear material behavior.

Slip pulse moving along a precut interface and generation of Mach waves. Classical fracture mechanics assumes that rupture extends in a monolithic, homogeneous linear elastic medium without any preexistence of other defects. If a precut interface like a geological fault plane exists in the medium and rupture runs along that interface, the upper limit of the rupture propagation speed may change. However, due to the fundamental difficulties in dynamic interface fracture mechanics, only a few theoretical studies have been conducted, and there is a deep disagreement even concerning the theoretically predicted propagation speed of interface rupture, e.g., slightly higher than the Rayleigh wave speed of the more compliant of the two dissimilar materials sandwiching the interface, ${ }^{46)}$ or the lower Rayleigh wave speed of the two contacting materials. ${ }^{47)}$ In the 
investigation of an edge dislocation moving along an interface at a constant velocity, it has been suggested that dynamic drop in compressive normal traction may allow a slip pulse to travel, along an interface governed by a constant coefficient of friction, in a self-sustaining manner near the $\mathrm{S}$ wave speed of the more compliant material. ${ }^{10), 48), 49)}$ Thus, vibration normal to an interface that propagates in a form of a pulse along the interface (hereafter, slip pulse) may be the key to understand "extraordinary" fast rupture propagation.

According to numerical simulations related to slip pulses, along a statically preloaded interface between similar materials waves involving separation of interface surface decay quickly rather than move in a self-sustaining manner ${ }^{50)}$ but slip pulses can be sustained if interface surface roughness is present ${ }^{51)}$ and slip pulses propagating in a self-sustaining manner may break up into a number of smaller pulses. ${ }^{49)}$ Experimentally, only several different types of laboratory investigations of dynamic rupture on precut interfaces have been performed for purposes of seismological study, but they do more clearly indicate that rupture can travel at the Rayleigh wave speed or higher. ${ }^{10), 52)}$ For instance, two-dimensional laboratory model experiments utilizing dynamic photoelasticity in conjunction with high-speed cinematography, together with finite difference numerical calculations, have illustrated that a slip pulse can run at the Rayleigh wave speed of the acoustically stiffer material and dynamic rupture-induced wave patterns are controlled by the acoustic mismatch of the two materials sandwiching the interface (Fig. 2a, b). Figure $2 \mathrm{a}$ depicts a slip pulse propagating from left to right along a statically preloaded, initially partially contacting interface between two identical transparent, birefringent linear elastic polymeric plates modeling a geological fault. Here, a snapshot of isochromatic fringe pattern (contours of maximum in-plane shear stress, $\tau_{\max }$; the fringe order is proportional to $\tau_{\max }$ ) taken by a dynamic photoelastic experiment is shown. In its leading right part, partial wave energy transmission occurs from the upper plate 1 across the interface into the lower plate 2 , but in the trailing left part, owing to the separational vertical particle movements from the interface in the plate 1 , the corresponding fringes in the plate 2 are missing, indicating the existence of dynamic interface slip (fault rupture). Indeed, this pulse involving interface slip propagates approximately at a Rayleigh wave speed, that is, dynamic rupture can travel at an "extraordinarily" high speed, $V / V_{S} \approx 0.9$. The isochromatic fringe pattern in Fig. $2 \mathrm{~b}$ obtained by a finite difference numerical simulation illustrates a slip pulse moving along an initially partially contacting interface, this time, between two dissimilar materials. In the beginning, the slip pulse is running at the Rayleigh wave speed of the acoustically stiffer material 1, $\left(V_{R}\right)_{1}$, which lies between the $\mathrm{S}$ and longitudinal $(\mathrm{P})$ wave speeds of the lower material 2, $\left(V_{S}\right)_{2}$ and $\left(V_{P}\right)_{2}$, i.e., $\left(V_{S}\right)_{2}<\left(V_{R}\right)_{1}<\left(V_{P}\right)_{2}$. The pulse energy is transferred along the preloaded, initially contacting region at an even more extremely high supershear speed with respect to the acoustically more compliant material $2\left(V /\left(V_{S}\right)_{2}>1\right)$, and as a consequence, a strong, energy concentrated Mach wave with high-frequency components is produced that propagates from the contacting region into the material 2. ${ }^{10)}$ Note that the pulse propagation speed with respect to the acoustically stiffer material 1 is subsonic also in this case but the same speed corresponds to a supershear one with respect to the material 2. This relatively high rupture propagation speed seems consistent with seismological observations, suggesting that in the linear elastic framework, there is an essential difference between rupture along an interface of a bimaterial system and that in a homogeneous monolithic medium.

If a slip pulse can propagate at depth in the Earth where very high compressive stress is expected to be applied, the existence of such a pulse, together with the universal nucleation length mentioned above, may be a solution to the earthquake-related paradoxes, e.g., origin of short rise times in seismic slip, ${ }^{53}$ anomalous $\mathrm{P}$-wave radiation, and low static shear stress levels on geological faults. ${ }^{49)}$ Moreover, such a pulse running in a bimaterial system and generating Mach waves may cause serious damage in a specific concentrated zone such as found in Kobe immediately after the January 1995 Hyogo-ken Nanbu, Japan, earthquake (moment magnitude $M_{w}=6.9$ ) (Fig. 2c): One of the puzzling phenomena having occurred in the Kobe region is the emergence of the "damage belt" or the strip of the most severely affected zones (the Japan Meteorological Agency Intensity 7 areas marked in red in Fig. 2c). This strip is located close, but not parallel, to the Suma/Suwayama faults where subsonic slip pulse is considered to have propagated at a shallow depth according to kinematic seismic inversion. ${ }^{54)}$ A region of relatively large slip, corresponding to the initially contacting region in the fault models in Figs. 2a and 2b, seems to be situated beneath the left edge of the strip, and a concentrated shear disturbance with large particle velocity was also 


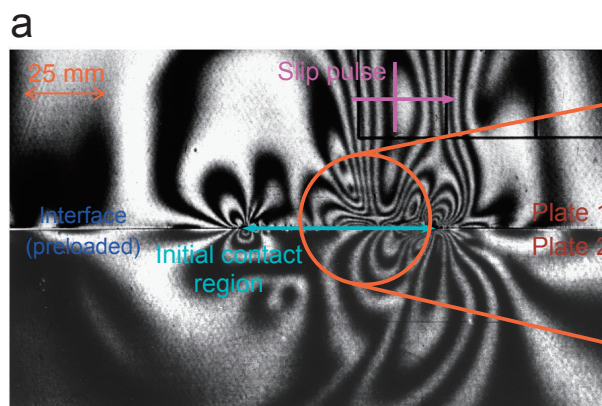

b
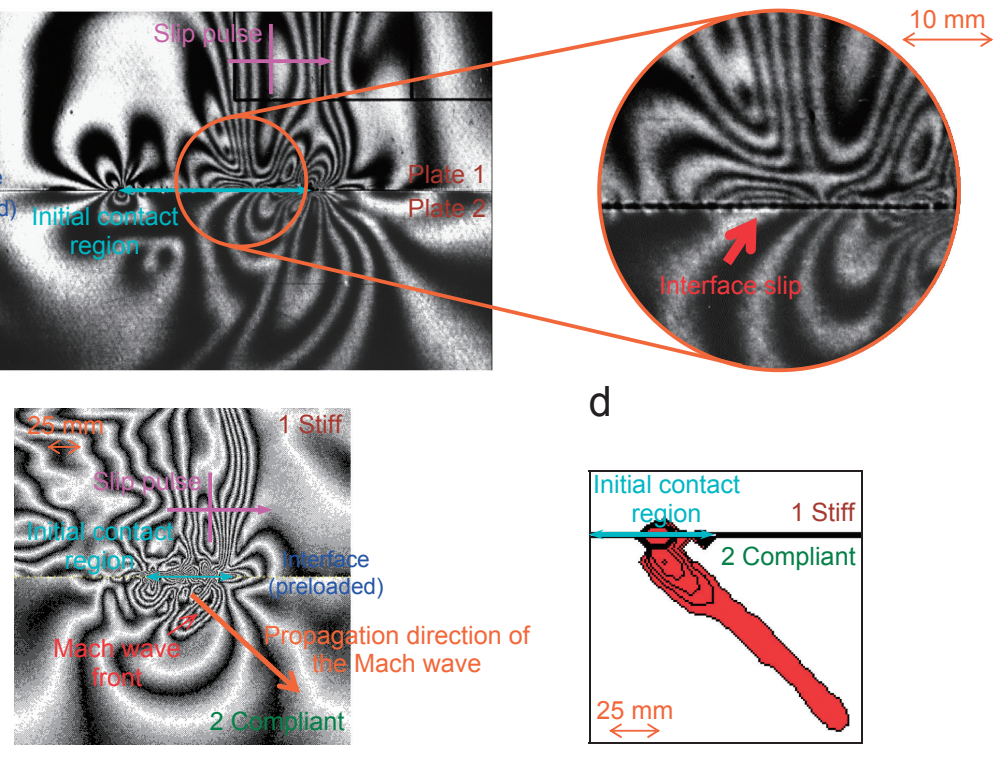

d

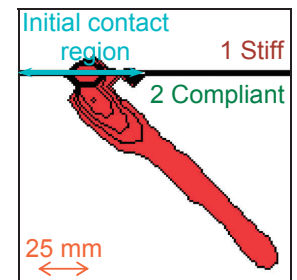

C
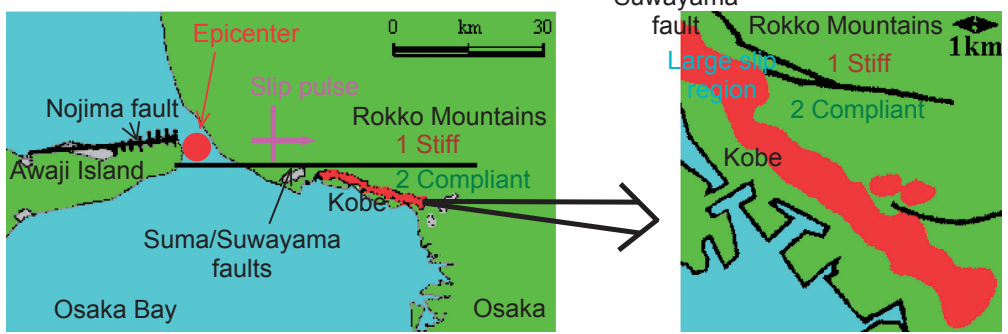

e
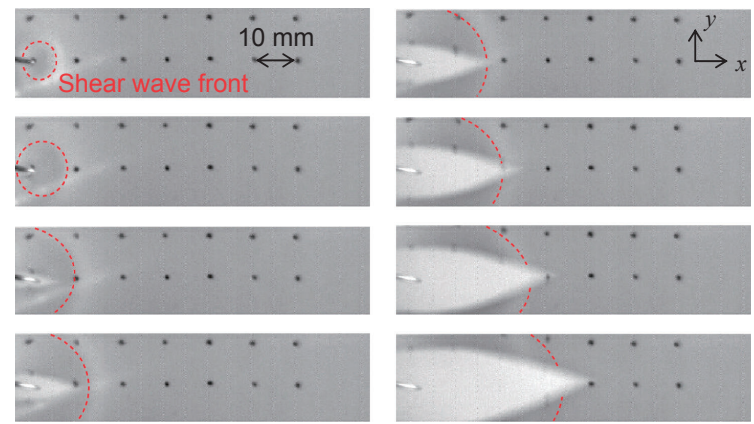

Fig. 2. (a) A slip pulse propagating along a statically preloaded, partially contacting interface between two identical polymeric plates that model a geological fault. Here, a snapshot of isochromatic fringe pattern (contours of maximum in-plane shear stress, $\tau_{\max }$ ) taken by a dynamic photoelastic experiment is shown. This pulse involving interface slip (fault rupture) moves at an "extraordinarily" high speed that is approximately equal to the Rayleigh wave speed or $90 \%$ of the shear (S) wave speed of the linear elastic plate. (b) If the interface is located between two dissimilar materials, the wave energy of the slip pulse initially traveling at the subsonic Rayleigh wave speed of the acoustically stiffer material 1 can be transferred along the initially contacting region at an even higher supershear (i.e., over the $\mathrm{S}$ wave) speed with respect to the acoustically more compliant material 2, and a strong Mach wave (sometimes called a "shock wave") with high-frequency components is generated in the material 2. (c) The mystifying "damage belt" found in the Kobe region just after the 1995 Hyogo-ken Nanbu, Japan, earthquake. The most severely affected areas are marked in red. (d) Due to the propagation of the energy-concentrated Mach wave in the more compliant material 2, the region of high peak particle velocity (PPV) for the dynamic rupture model (b) is located in a narrow band, which is similar to the shape of the damage belt in (c). ${ }^{10)}$ (e) Fast rupture propagation process in a biaxially stretched hyperelastically deforming rubber sheet, recorded by a high-speed digital video camera system. The rupture front moving from left to right in this nonlinear elastic material captures the shear wave front indicated by red broken lines, clearly indicating that dynamic rupture can accelerate to a supershear speed even without existence of precut interfaces. Elapsed time after rupture initiation by pricking the sheet is $0,41.7,83.3,125.0,166.7,208.3,250.0$ and $291.7 \mu$ s, respectively. ${ }^{58)}$ 
recorded in central Kobe. ${ }^{54}$ This implies that a Mach wave was induced by fault rupture in the acoustically more compliant downtown area. Damage due to liquefaction was hardly observed inside this severely affected area, and therefore it is suggested that the damage was caused directly by the seismic waves. ${ }^{10), 55)}$ During the course of such dynamic wave interaction, each particle in materials experiences a history of velocity, and its maximum value for every particle is of practical importance. Hence the peak particle velocity (PPV) has been selected as the design parameters in engineering applications. For the dynamic rupture model in a bimaterial system in Fig. 2b, apparently because of the propagation of the strong Mach wave in the acoustically more compliant material 2, the shape of the region of high PPV (Fig. 2d; the zone of particles with absolute values of PPV over $85 \%$ of the maximum one) becomes similar to that of the damage belt in Kobe (Fig. 2c). The model material 1 fits the acoustically stiffer area in the foothills of the Rokko Mountains where soils are very shallow or rock outcroppings prevail and the damage by subsonic slip pulse propagation tended to be relatively minor. The material 2 corresponds to the acoustically more compliant region in which primarily soft alluvial soils are found and the slip pulse, moved at a supershear speed locally, induced a Mach wave and the damage belt. ${ }^{10)}$

Fast rupture propagation in nonlinear elastic materials. Exceptionally, as stated above, several different types of laboratory experiments of dynamic rupture on precut interfaces have indicated the existence of dynamic rupture moving at the Rayleigh wave speed or higher, but the inconsistencies of rupture speeds between theories, seismological observations and experiments (and numerical simulations) cannot always be attributed to the microscopic observations of materials that real solids have all kinds of defects such as preexisting discontinuities or microcracks generated during rupture propagation, because similar discrepancies may also emerge in molecular dynamics simulations of rupture running in perfect atomic lattices. Large-scale atomistic simulations ${ }^{56)}$ have suggested that nonlinear elasticity of large strains, nonlinear hyperelastic stressstrain relation in solids, may govern the dynamics of brittle fracture and it may, instead of widely assumed linear elasticity, resolve the contradictions. Real solids like the crust, indeed, have elastic characteristics that are notably dissimilar at various scales, for both small and large deformations. While quasistatic loading may give crustal rocks nonlinear strain- softening effect (stress reduction after a peak value with increasing strain), hyperelastic rubber-like strain-hardening behavior of rocks (nonlinear increase of stress with strain) may prevail under dynamic loading, e.g., due to detonation by blasting. ${ }^{57)}$ The experimental investigation into mode I tensile rupture initiation and dynamic propagation process in hyperelastically deforming rubber sheets with a high-speed digital video camera system has shown that the dynamic rupture characteristics depend more strongly on the static loading conditions in the nonlinear hyperelastic case than in the linear elastic one. If the static normal uniform stress (crackparallel or $T$-stress) acting parallel to the rupture propagation direction ( $x$-direction in Fig. $2 \mathrm{e}$ ) is of the same magnitude compared with the remote tensile loading stress in the $y$-direction that drives rupture propagation in the $x$-direction, the rupture travels astonishingly straight and, even without the preexistence of material inhomogeneities, the rupture front accelerates from zero to a constant supershear speed to form a very sharp wedge shape and capture the shear wave front produced upon rupture initiation (Fig. 2e). The rupture speed often satisfies $V / V_{S}>2$ and the seismologically estimated value $\left(V / V_{S} \approx 1\right)$ is, at least from a viewpoint of hyperelastic mode I rupture, not extremely high. ${ }^{58)}$ This is completely different from the above interpretation of fault rupture where precut interfaces between similar or dissimilar materials are assumed so as to achieve the "extraordinary" high rupture speed. In any event, the observations outlined in this chapter may possibly render a new hint on the question of whether natural earthquake ruptures can really dynamically accelerate to such "extraordinary" high speeds as have been inferred from kinematic seismic inversions.

\section{Underground failures and seaquakes: effect of higher-frequency seismic waves with vertical oscillations}

So far, fundamental mechanisms of earthquake nucleation, rupture propagation and wave radiation have been addressed. Traditional engineering seismology presumes the dominant role of low-frequency horizontal vibration in producing structural failures, but in this and the following chapters, possible significant influence of higher-frequency components of seismic waves that are moving in the underground and vibrating vertically or horizontally at surface (at sea) will be summarized by treating the dynamic connection between seismically radiated waves and "unusual" or "unexpected" structural behaviors. It 
may be possible to "inversely" evaluate the mechanical characteristics of the seismic waves not only conventionally from seismograms but also from the associated structural failure patterns.

Note, first of all, that in a lower frequency range, as traditional analyses presume, horizontal vibrations may be dominant than vertical ones in the seismological records, but near an epicenter, like in Mashiki Town (KMMH16 station of the Japanese strongmotion seismograph network of the National Research Institute for Earth Science and Disaster Resilience (NIED), KiK-net, epicentral distance $2 \mathrm{~km}$ ) during the April 16, 2016, Kumamoto main shock $\left(M_{w}=7.0\right)$, vertical up-down movement may become more dominant than the horizontal one in a higher frequency range (over some $3.7 \mathrm{~Hz}$ at KMMH16). As described above, also the 1995 Hyogoken Nanbu earthquake may have generated highfrequency seismic waves. Unfortunately, however, the sensitivity of the SMAC-MDU strong-motion accelerometers (accelerographs) commonly deployed at that time dropped drastically above $20 \mathrm{~Hz}$ (usually for technical reasons) and waves over $10 \mathrm{~Hz}$ were filtered out, and therefore, it was difficult for them to detect higher frequencies. ${ }^{59)}$

"Unusual" damage to underground facilities. Although failures of underground facilities owing to an earthquake hardly occur, in the 1995 seismic event, the Bantaki Tunnel in the Rokko Mountains has suffered unique structural failure (Fig. 3a). The failure was found in the central section of the tunnel that had been completed about four years before the quake utilizing the New Austrian Tunneling Method (NATM). On the sidewall, dynamic compression seemed to have caused exfoliation of lining concrete and buckling of reinforcing steel bars. In addition, the subgrade detached (some $10 \mathrm{~cm}$ ) from the invert, but the invert itself was not deformed. No damage existed at the crown (on the ceiling), either, and no distinctive sign of permanent ground deformation was observed at the failure site. ${ }^{60}$ ) Since the site was located in the middle section of the approximately $1,750 \mathrm{~m}$-long tunnel with the rather large overburden thickness about $250 \mathrm{~m}$ and no permanent ground deformation existed, the failure may have been generated not by fault displacement or instability near the portal, but by the dynamic diffraction of seismic body waves around the tunnel. Similar structural failure patterns ${ }^{61)}$ were found in the Uonuma Tunnel of the high-speed Shinkansen train network just after the 2004 Niigata-ken Chuetsu, Japan, earthquake $\left(M_{w}=6.6\right)$.
For the essential comprehension of wave diffraction and scattering by structural inhomogeneities such as cavities (tunnels) and inclusions, the timeharmonic analytical elastodynamic approach based on the wave function expansion method has been repeatedly adopted. Typical problems are scattering of an incident plane harmonic $\mathrm{P}$ wave by a circular cavity ${ }^{62)}$ an arbitrarily thick elastic cylinder ${ }^{63}$ ) and a group of cylinders in parallel ${ }^{64)}$ in an infinitely extending elastic medium. In the same way, dynamic response of a cylindrical cavity or rigid inclusion caused by incident harmonic in-plane shear (SV) wave $^{65)}$ has been analyzed, and together with the image technique for the reflection of the waves at the ground surface, the incidence of an anti-plane shear (SH) wave on a single ${ }^{66)}$ or twin ${ }^{67)}$ circular tunnel(s) has been considered. These analyses do not seem to have been applied to real dynamic problems of failures of underground facilities, but, if a similar two-dimensional time-harmonic elastodynamic approach is utilized and diffraction of plane harmonic $\mathrm{P}$ (vertical oscillations) and SV (horizontal vibrations) waves by a simplified, uniformly lined circular tunnel situated in an infinitely extending linear elastic medium is analytically evaluated, it can be systematically shown that only $\mathrm{P}$ waves in a relatively high frequency range may be able to induce dynamic compression on the sidewall, impart large accelerations to the bottom of the circular tunnel, and as a result produce the failure pattern like that observed in the Bantaki Tunnel (Fig. 3b). ${ }^{59)}$ For example, the stress concentration factor, or the ratio of the dynamic maximum compressive stress $\left|\left(\sigma_{\theta \theta}\right)_{I}\right|$ to the stress amplitude of the incident body wave $\sigma_{0}$, $\left|\left(\sigma_{\theta \theta}\right)_{I} / \sigma_{0}\right|$, plotted for the sidewall $\left(|\theta|=90^{\circ}\right)$ of an unlined tunnel (radius $a=b$ ) in Fig. $3 \mathrm{~b}$, indicates that for standard earthquakes where the wavelength of an associated wave $\left(\left(\lambda_{P}\right)_{I}\right.$ for $\mathrm{P}$ or $\left(\lambda_{S}\right)_{I}$ for $\mathrm{SV}$ wave) is considered to be much longer than the dimension of a tunnel, say, $\left(\lambda_{P}\right)_{I} / a,\left(\lambda_{S}\right)_{I} / a \geq 10$, the horizontal $(\mathrm{SV})$ disturbances cause stress reduction $\left(\left|\left(\sigma_{\theta \theta}\right)_{I} / \sigma_{0}\right|<1\right)$ while the vertical (P) oscillations may give stress concentration $\left(\left|\left(\sigma_{\theta \theta}\right)_{I} / \sigma_{0}\right|>2\right)$ on the sidewall. For the wavelengths $\left(\lambda_{P}\right)_{I} / a=\left(\lambda_{S}\right)_{I} /$ $a=50$, the stress concentration factors are $\left|\left(\sigma_{\theta \theta}\right)_{I}\right|$ $\sigma_{0} \mid \approx 2.8$ and $\left|\left(\sigma_{\theta \theta}\right)_{I} / \sigma_{0}\right| \approx 0.17$ for $\mathrm{P}$ and $\mathrm{SV}$ waves, respectively. That is, if some rupture criteria based on compressive stresses may be applied, $\mathrm{P}$ waves with much smaller amplitudes (about $6 \%$ of those needed for SV waves) can generate compressive damage on the sidewall. Note also that no vertical accelerations and therefore no damage such as 
a
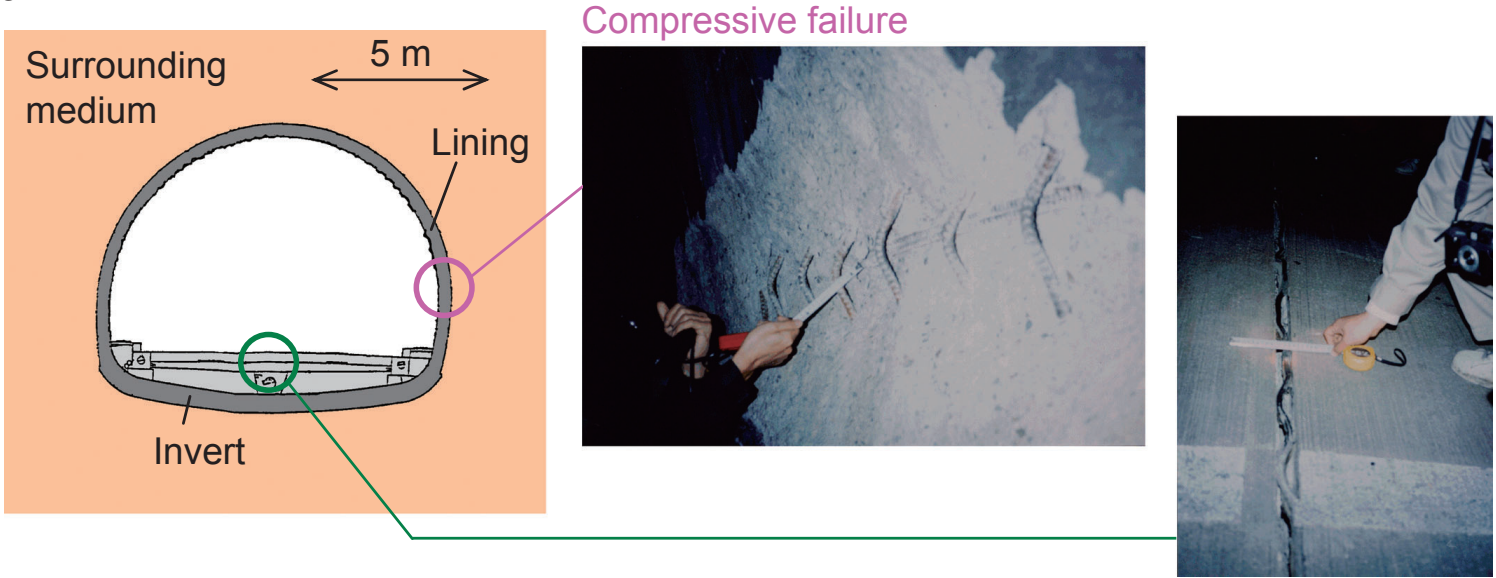

Subgrade detachment

b
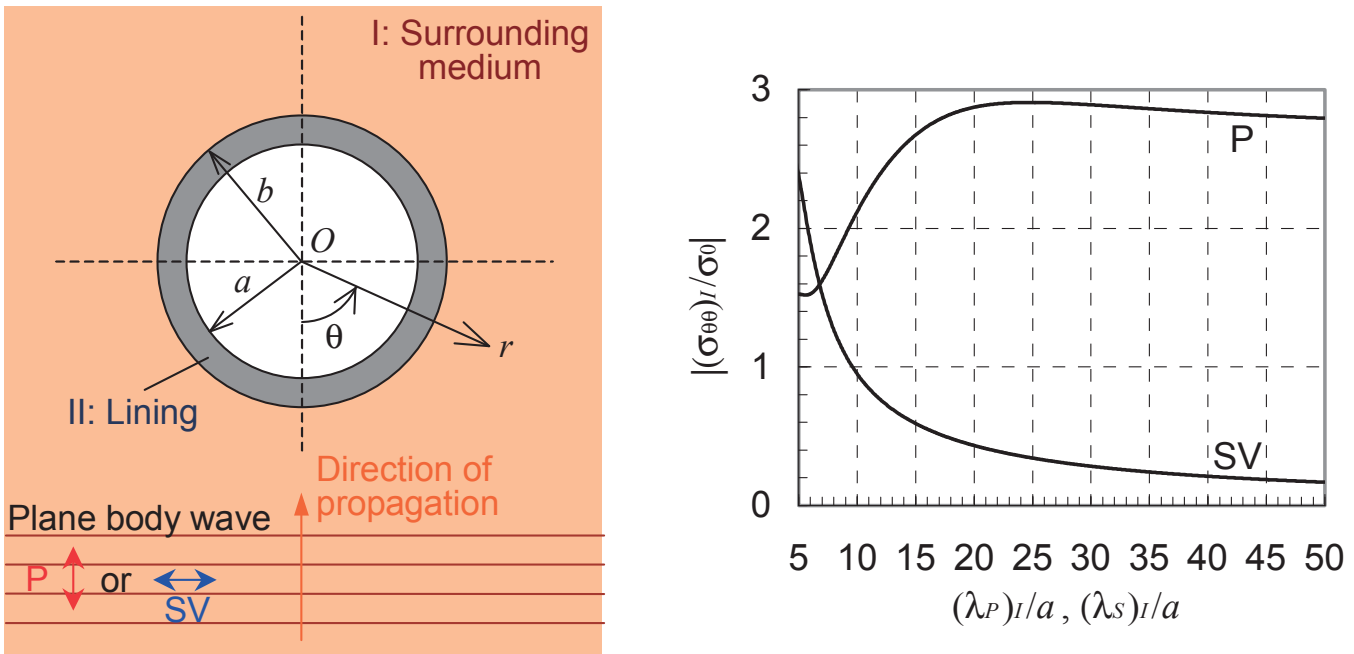

C
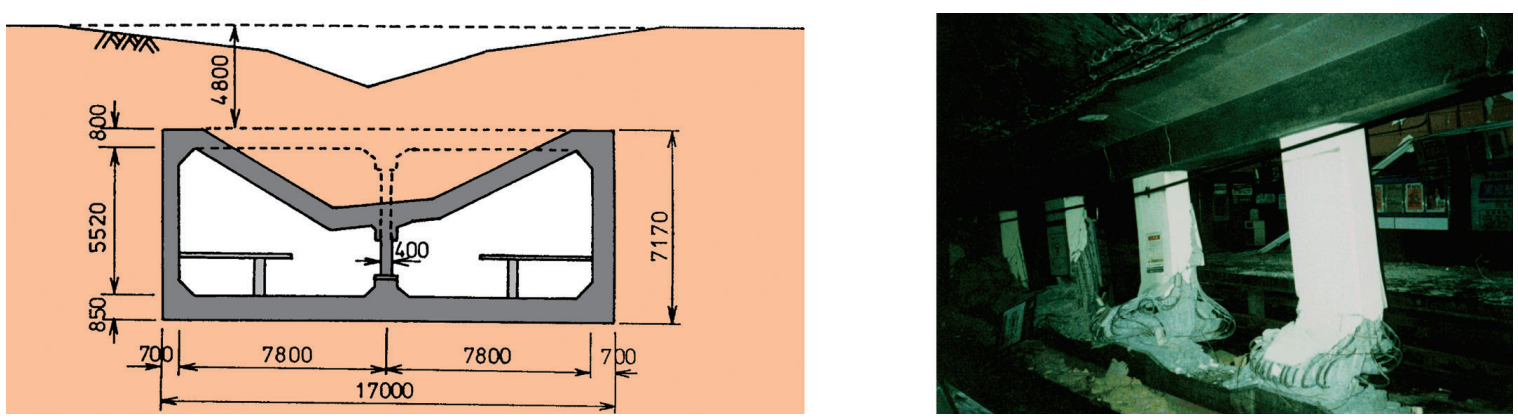

Fig. 3. (a) "Unusual" structural failure in the Bantaki Tunnel generated by the 1995 Hyogo-ken Nanbu earthquake (cross-sectional view). (b) Diffraction of plane time-harmonic vertical ( $\mathrm{P}$ wave) or horizontal (SV wave) disturbances by a simplified tunnel located in an infinite, homogeneous linear elastic medium. ${ }^{59)}$ (c) A typical cross-section of the Daikai underground station in Kobe after the 1995 quake (left; all units in $\mathrm{mm}$ ). Failure of the columns supporting the roof at midspan (central columns) gave rise to the vertical collapse of the roof (right). A simple dynamic analysis suggests that it is more straightforward to regard also this failure as a phenomenon caused by vertical vibrations in a higher frequency range, over $10 \mathrm{~Hz}^{68)}$ (Photographs courtesy of Professor Emeritus Shunsuke Sakurai of Kobe University) 
subgrade detachment may be expected at the bottom of the tunnel $\left(|\theta|=0^{\circ}\right)$ by incidence of SV waves. Similarly, it can be demonstrated that in the Bantaki Tunnel, incident $\mathrm{P}$ waves consisting of higherfrequency components were diffracted at the bottom and there the particle acceleration was amplified about twice to result in the detachment of the more accelerated subgrade. On the ceiling of the tunnel $\left(|\theta|=180^{\circ}\right)$, however, the higher-frequency components had already attenuated and produced no damage. This is not the case for low-frequency $\mathrm{P}$ waves that give more or less the same magnitude of particle accelerations at the bottom and on the ceiling of a lined tunnel. The failure in the Bantaki Tunnel, together with another underground failure pattern found in Kobe in 1995, the collapse of the reinforced concrete columns at the Daikai underground station (Fig. 3c), ${ }^{60), 68)}$ suggests that structures subjected to seismic waves may well function as "sensors" that detect and respond only to waves of particular characteristics, and this time, "unusual" (in a conventional sense) vertical oscillations in a higher frequency range (over $10 \mathrm{~Hz}$ ) seem to have impinged upon them. ${ }^{59)}$

Seaquakes: vertical shocks experienced at sea. Besides the "unusual" structural failures observed in the underground, another unique phenomenon, "seaquake," was reported in the Kobe region in 1995. Seaquakes are strong vertical shocks felt on board a floating body (e.g., ship, vessel) at sea during a seismic event, and they may be sufficiently violent to cause serious damage to, or, like the case of the schooner "Henrietta" in the North Sea in 1894, ${ }^{69)}$ even sinking of, floating bodies. Unlike tsunamis, however, seaquakes are usually experienced only near the epicenter of an earthquake. ${ }^{69)}$ Thousands of occurrences of seaquakes are recognized ${ }^{69)-75)}$ but only a very limited number of them are listed in the casualty reports like Lloyd's List. ${ }^{72)}$

In the 1995 Hyogo-ken Nanbu case, no less than four ferry boats, whose positions depicted in Fig. 4a, were hit by seaquakes. The captains of the 9,000tonne "Queen Diamond" and the 10,000-tonne "Sunflower" felt two violent vertical shocks, with the interval between each shock being 1 or $2 \operatorname{second}(\mathrm{s}){ }^{73)}$ In the instance of the ferry boat "Asagirimaru" that had become out of control due to the first impact, the interval between the two shocks was longer, a few minutes, ${ }^{75)}$ while the 15,000-tonne "New Nagato," located farther from the epicenter, was struck only by one vertical shock, which was absolutely without horizontal shaking. According to the ultrasonic wave a

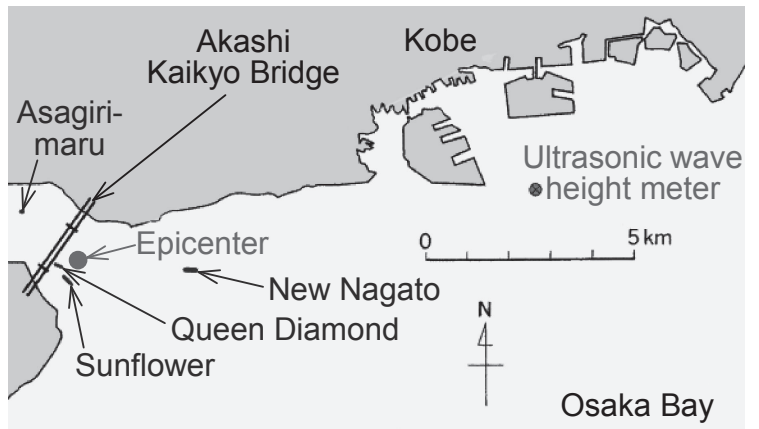

b

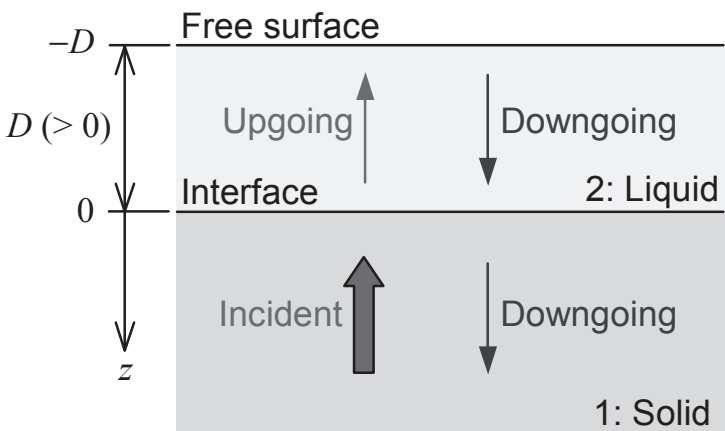

C

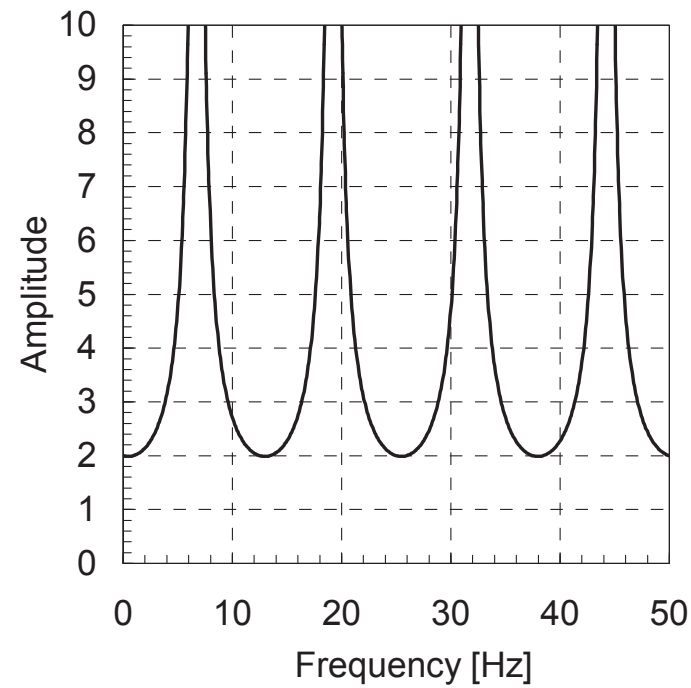

Fig. 4. (a) Locations of the four ferry boats on which seaquakes, vertical shocks at sea, were felt during the 1995 Hyogo-ken Nanbu earthquake. (b) A really simple one-dimensional elastodynamic model where wave interaction at the solid-liquid interface as well as that at the stress-free surface are considered. A liquid layer (seawater) of finite thickness exists on top of the semi-infinitely extending solid (seabed). (c) Amplification of the amplitude of the vertical particle displacement (velocity, acceleration) at the seawater surface for the 1995 "seaquake" in Kobe. It is significantly dependent on the frequency of the incident $\mathrm{P}$ wave in the seabed. ${ }^{80}$ 
height meters near the Port of Kobe and the OsakaKansai International Airport, tsunamis were only some $5 \mathrm{~cm}$ high and they were without any two clear peaks. ${ }^{73)}$ Obviously, seaquakes are different from tsunamis.

From a physics point of view, seaquakes are often linked to propagation of $\mathrm{P}$ acoustic waves through water. ${ }^{69)}$ But their generation mechanisms have not been completely understood yet, and this gives major concern to mariners, engineers and scientists who examine the safety and security of naval architecture and offshore structures. In the study of seaquakes, normally, only hydrodynamic behavior of potential flow in seawater is investigated with the assumption of the rigid seabed (like a "shaking table" in conventional methods of engineering seismology) and no dynamic interaction between seawater and the seabed. ${ }^{76-79)}$ However, a simplified one-dimensional dynamic analysis of linear elastic solid-liquid interaction (between the semi-infinitely long seabed and a layer of seawater of finite thickness) as well as nonlinear vertical shock wave propagation has suggested two possible seaquake generation mechanisms: (a) quick resonance of the solid-liquid system in a higher frequency range (at some $6.7 \mathrm{~Hz}, 19.2 \mathrm{~Hz}, \ldots$ for the 1995 case; Fig. 4b, c); and (b) slow development and propagation of a nonlinear wave of velocity discontinuity (shock) in seawater that is associated with the Burgers' equation in fluid mechanics. The combination of these two temporally different mechanisms, both connected to higher-frequency wave components, may enable us to explain why seaquakes may be sometimes felt twice at some time intervals during a single seismic event. ${ }^{80)}$

\section{Tensile slope failure and surface waves}

In the above examples of the "inverse" analyses of the dominant seismic waves from the related structural failure patterns in the Kobe region, mainly body waves have been treated and the crucial role of higher-frequency vertical vibrations has been addressed. Now the possible influence of another type of elastic waves, surface waves that induce both vertical and horizontal movements at surface, will be pointed out with "unfamiliar" unique tensile failures in slopes and cliffs found after several seismic events (Fig. 5).

Although much more attention has been paid to tsunamis and their devastating effects on structures than the influence of seismic waves, the off the Pacific coast of Tohoku earthquake in March 2011 has affected innumerable structures severely in the Tohoku and Kanto regions of Japan. One noteworthy structural failure may be the one in the fill slope in Sendai where the quake $\left(M_{w}=9.0\right)$ generated crack openings (Fig. 5b) on the top surface along the upper edge (crest). The vital facts here are that in the same slope very similar vertical tensile cracking was recognized along the crest by the 1978 Miyagi-kenoki earthquake $\left(M_{w}=7.5\right)$ (Fig. 5a) and the slope was reinforced with steel pipe piles after this 1978 event using conventional countermeasures that take into account mainly the effect of $\mathrm{S}$ waves (often replaced by horizontal vibrations, as described below). ${ }^{81 \text { ) }}$

Besides these important facts, it should also be noted that tensile, open cracks of the same type were observed in California, the United States, after the $1906\left(M_{w}=7.8\right)$ and $1957\left(M_{w}=5.7\right)$ San Francisco $^{82}$ and the 1989 Loma Prieta $\left(M_{w}=\right.$ $6.9)^{83)}$ earthquakes as well as in the South Island, New Zealand, on the occasion of the February 2011 Christchurch earthquake $\left.\left(M_{w}=6.2\right) .{ }^{84}\right)$ The slopes (cliffs) in these cases are larger in scale than the Japanese one, up to the height of $50 \mathrm{~m}$ in California. According to the photographs of the two San Francisco earthquakes, the (nearly) vertical slopes in the weakly cemented sands experienced disconnection of a narrow block of soil along the vertical tension crack on the top surface of the cliff and later shearing at the bottom of the block. ${ }^{82)}$ The 1989 quake generated tension cracks extending 1 to $6 \mathrm{~m}$ behind the crests of coastal bluffs. ${ }^{83)}$ In the New Zealand case, photographs ${ }^{84)}$ show that the cracks are located in a rather monolithic, homogeneous medium and do not seem to run along some specific geological planes of weakness.

A "realistic" way of including seismic effects in the assessment of slope failures is to assume a slope made of some deformable materials on a rigid "shaking table" (or with an energy-absorbing bottom) that is subjected to uniform, prescribed horizontal vibration (replacing plane $\mathrm{SH}$ or SV wave incidence). Motivated in part by the observations of the dynamic failures in California, the response of steep slopes subjected to vertically moving horizontal vibrations ${ }^{82), 85)}$ or inclined plane $\mathrm{S}$ waves ${ }^{83)}$ has been numerically investigated. But unfortunately, these numerical simulations, mainly analyzing the accelerations at the crest, do not seem to explicitly provide quantitative information about the precise reason and exact positions of open cracks. ${ }^{86}$ ) That is, analyses treating only the interaction of body (S) waves cannot entirely describe the dynamic slope behavior.

In the 1978 Sendai case, seismic waves generated open cracks at a relatively small distance, some 6 
a

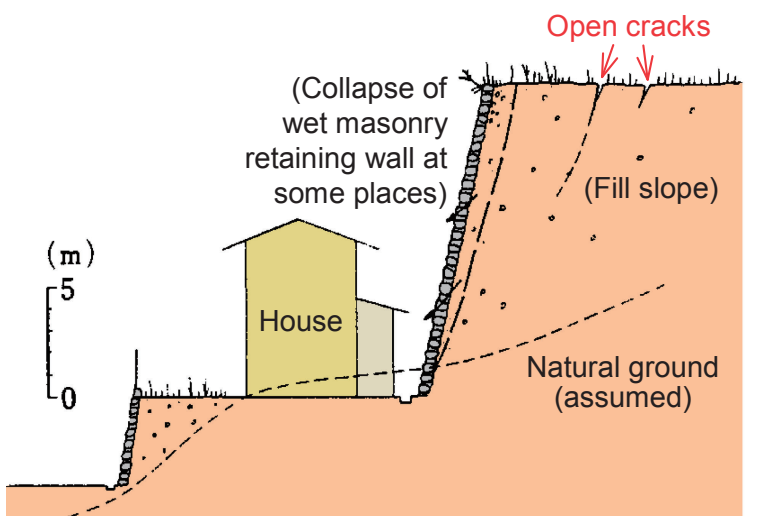

C

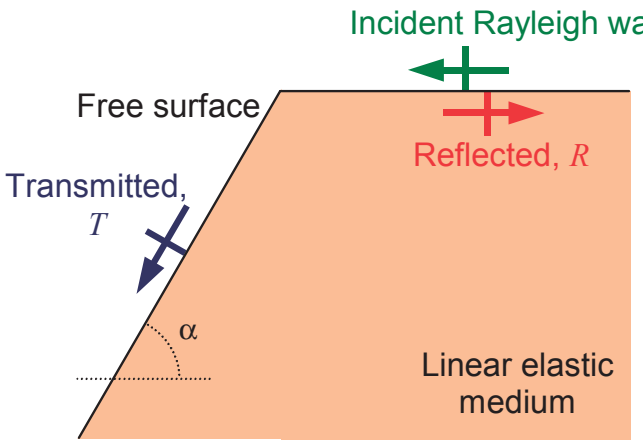

b

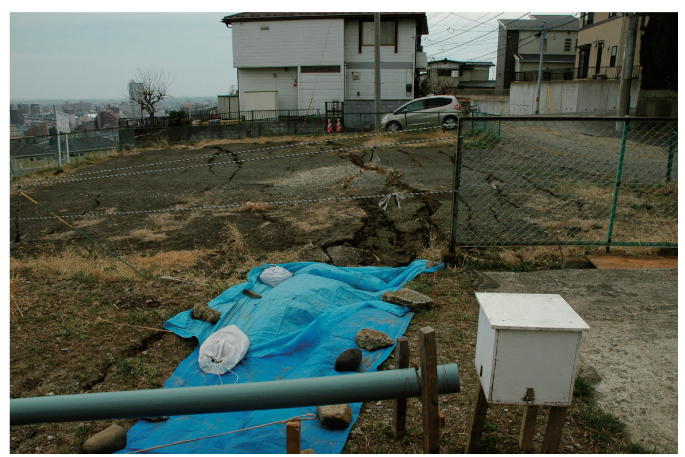

d

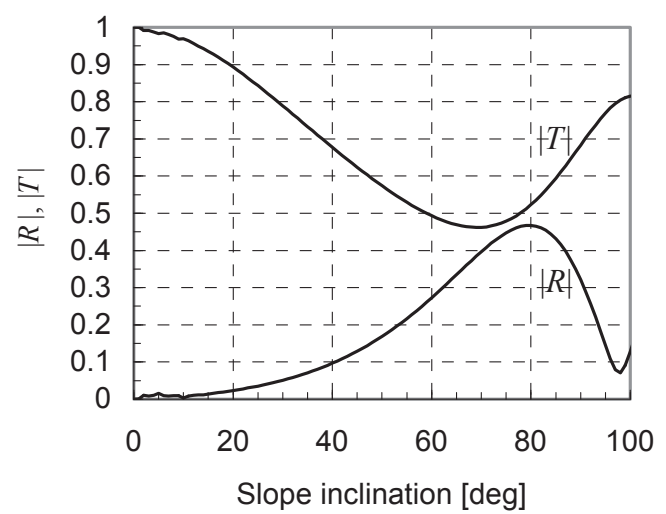

e
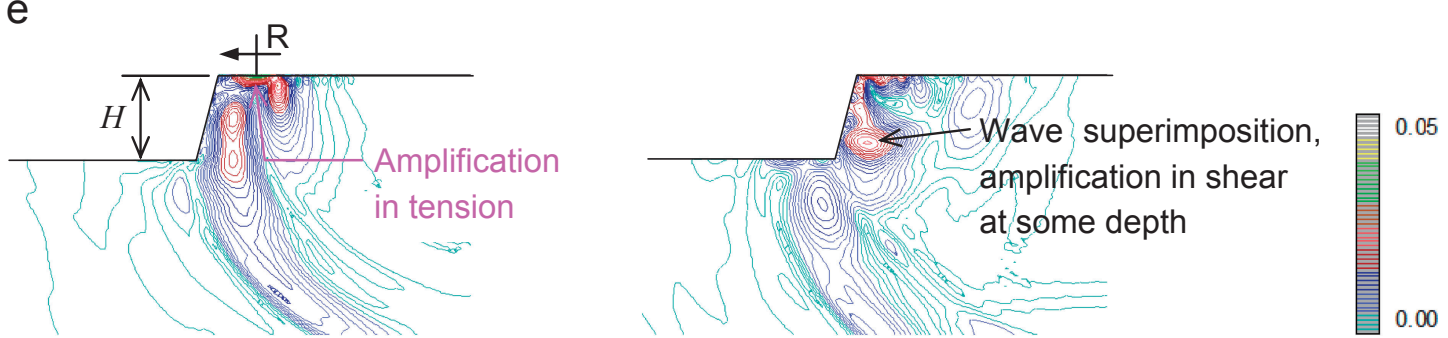

Fig. 5. (a) Typical failure of a fill slope in Sendai, observed after the 1978 Miyagi-ken-oki, Japan, earthquake. ${ }^{86)}$ (b) Tensile cracking along the upper edge (crest) of the same (but reinforced after 1978) slope on the occasion of the 2011 off the Pacific coast of Tohoku earthquake (Photograph courtesy of Professor Nobuyuki Torii at Kobe City College of Technology). (c) The interaction of the incident Rayleigh surface wave with the crest of a convex linear elastic slope generates reflected and transmitted Rayleigh waves as well as body waves moving to the far field. (d) The dependency of (the absolute values of) the reflection and transmission coefficients, $|R|$ and $|T|$, on the slope inclination $\alpha$ for the case Poisson's ratio 0.25 indicates that $|R|$ becomes maximum at $\alpha=80^{\circ}$ while $|T|$ is at its minimum at $\alpha=69^{\circ}$. That is, around $\alpha \approx 70-80^{\circ}$ (like in the Sendai case), at the top surface the superimposed (incident plus reflected) wave amplitude may become about 1.5 times the incident one or three times as large as the transmitted one, and tensile cracking is more likely to be induced on the top surface than in the slope face (if a tensile stress criterion can be applied). Note that Rayleigh waves in a monolithic two-dimensional medium are nondispersive, i.e., these results do not depend on wave frequencies, but frequencies (or wavelengths) do play a role in determining the exact positions of strong stress amplification due to the superimposition. (e) A typical example of the full numerical MPS simulations of a dynamic Rayleigh surface wave propagating from right to left along a free surface and interacting with a more realistic model slope (inclination angle $75^{\circ}$ ). The development of the normalized maximum in-plane shear stress $\tau_{\max }$ is shown for the incidence of a higher-frequency wave where the wavelength is of the order of the slope height $H{ }^{81)}$ The slope investigated is facing generally towards NNE and the epicenter is roughly located to the ESE of the failure site in Sendai. Therefore, it is possible that surface waves arrive, like in this simulation, at the upper level of the slope and propagate down along the face to the lower level. 
meters, from the crest (Fig. 5a). The slope inclination angle was nearly vertical, approximately 75 degrees, and except for the collapse of wet masonry retaining walls at some places, no damage was recognized in the slope face and no clear circular slip surface was found at the failure site. These data imply that waves in a relatively higher frequency range, i.e., wavelength comparable to the height of the slope, interacted with the slope to induce dynamic tensile stresses at the top surface of the slope near the crest. Because of the relatively large epicentral distance over $100 \mathrm{~km}$, the failure may have been owing to Rayleigh surface waves, with less attenuation $\left(\sim 1 / r^{1 / 2}\right)$ than body waves $\left(\sim 1 / r^{2}\right.$ along the surface; $\sim 1 / r$ otherwise) at a distance $r$ from a surface source. Hence, in order to explain dynamically the generation mechanism of the slope failure in a systematic way, two-dimensional interaction of a time-harmonic Rayleigh surface wave with a simple, linear elastic slope has been quantitatively evaluated using the method of Fourier transformations (Fig. 5c). ${ }^{86}$ ) The results, the semianalytically calculated reflection and transmission coefficients, $R$ and $T$ that are defined as the ratios of the complex displacement amplitudes of the reflected and transmitted Rayleigh waves to that of the incident wave and plotted as functions of the slope inclination angle $\alpha$ (Fig. 5d), suggest that the angle 75 degrees like in Sendai is not especially appropriate for linear elastic slopes subjected to Rayleigh wave incidence: The crest may serve as an efficient wave amplifier at the top surface, where more energy is reflected and the superimposition of the incident and largely reflected Rayleigh waves may give strong (tensile) stress amplification to initiate open cracks at a particular position from the crest. If the slope behavior in Sendai may be regarded as linear elastic, the frequency of the incident Rayleigh wave is theoretically estimated to be approximately $10 \mathrm{~Hz}$ in order to cause open cracks at the position about $6 \mathrm{~m}$ from the crest. In other words, if a Rayleigh wave has produced this failure, the surface wave should carry, as mentioned, relatively higher frequencies. The coefficients $R$ and $T$ are strongly dependent on the inclination angle $\alpha$, and if the slope were less steep, say, $\alpha \approx 60^{\circ}$, the reflected wave amplitude would decrease by $40 \%$, and hence less damage to the top surface would be expected.

The above possible fundamental role of higherfrequency Rayleigh surface waves in the generation of tensile cracking may be confirmed, at least in terms of stresses, by two-dimensional numerical simulations for non-harmonic body or Rayleigh wave interaction with an archetypal linear elastic slope using the original version of the moving particle semi-implicit (MPS) method. ${ }^{81)}$ The development of the normalized $\tau_{\max }$ induced by dynamic Rayleigh wave-slope interaction (Fig. 5e) shows that, first, as expected from the semi-analytical investigation, large amplification in tension because of the superimposition of the incident and reflected Rayleigh waves can be recognized on the top surface at a relatively small distance from the crest (left snapshot). This amplification in tension, however, vanishes very quickly and at normalized time $V_{R} t / H=0.638$ later ( $t$ : time, $H$ : slope height), the latter part of the incident Rayleigh wave is superimposed with the transmitted first part and $\tau_{\max }$ is amplified at some depth from the top surface (right). Thus, crack opening on the top surface (by the tensile stress) and subsequent slope destabilization (in shear at some depth like observed in California) may be caused due to the action of a higher-frequency Rayleigh surface wave. However, MPS simulations of dynamic interaction of $\mathrm{P}$ and SV body waves with the same model slope cannot reproduce such failure patterns, even in a higher frequency range. ${ }^{81)}$

The presence of the theoretically estimated "unusual" higher-frequency wave (some $10 \mathrm{~Hz}$ ) for the epicentral distance over $100 \mathrm{~km}$ might be suspicious. However, the Sendai region is historically known as one of the places on the eastern seaboard of northern Japan where the subducting Pacific Plate functions as an efficient waveguide for high frequencies and these may be conveyed to the surface through scattering by inhomogeneities in plate structure, ${ }^{87}$ ) with possible conversion of body waves into Rayleigh surface waves. The velocity response spectra also seem to reinforce the idea that high-frequency seismic waves did exist in Sendai during the $1978^{86)}$ and the March 2011 earthquakes (see e.g., the velocity response of the MYG013 station recorded for the 2011 event by the K-NET of NIED). It may be possible to mention, at least, that for a systematic explanation of the generation mechanism of open cracks on the top surface at such a small distance from the crest, analyses should include not only the propagation of body waves but also the incidence of Rayleigh surface waves in a relatively higher frequency range. ${ }^{81)}$

\section{Town effect: seismic waves radiated from surface structures}

In the previous cases, the structural failures were possibly caused by "unusual" waves with vertical 
oscillations. But here, it will be shown that even "traditional" body waves with horizontal vibrations can induce "unexpected" serious damage when they interact with an assembly of structures standing close to each other.

In the conventional models for describing seismic performance of structures in a town or a city, it is normally assumed that one structure consisting of rather realistic elements (and again, usually standing on a rigid "shaking table") is subjected to seismic oscillations, and the vibrational response of that individual structure is separately examined in detail. That is, although numerous structures exist densely in towns or cities, the interaction of the (vibrations of) structures with the waves in the ground (hereafter, dynamic coupling effect) is often ignored in these analyses. Obviously, in the situation where multiple structures are constructed closely in a small area, the coupling effect will not be neglected. The dynamic coupling effect drew much more attention after the 1985 Michoacan earthquake $\left(M_{w}=8.0\right)$ that had caused severe damage to Mexico City. The difficulties of ordinary numerical methods in matching the seismological records have provided an idea that some portion of the seismic energy transmitted to surface structures may be radiated back into their neighborhood through the dynamic coupling effect. This phenomenon is sometimes named "site-city interaction," and the subsequent computations with different mechanical models of a "city" tend to have supported the concept. ${ }^{88)-94)}$ Unfortunately, so far, accurate vibrational behavior of every structure in a city has not been fully identified yet, but a snapshot taken after the 1976 Friuli earthquake, historically one of the greatest ones in Italy $\left(M_{w}=6.5\right)$, is claiming that the dynamic coupling effect should not be discarded in reality. Figure 6a, showing an amazingly periodic structural failure pattern in the epicentral Gemona region, suggests that, even if the importance of the causes such as the vulnerability (toughness) of each individual structure is accepted, it is not simple to systematically explain the generation of the alternate damage levels occurring in such a small spatial distance: If the structures built next to each other are subjected to more or less the identical (dominant types and frequency components of) seismic waves under very similar geological conditions, the structural damage should be, to some extent, also comparable.

The mechanical effect of dynamic coupling in a town containing multiple surface structures (buildings) can be evaluated quantitatively by performing a fully-coupled two-dimensional elastodynamic analysis with a simple model where each building in the town is replaced by a rigid foundation, a mass at the top and an elastic spring that connects the foundation and mass. ${ }^{94)}{ }^{95)}$ In the model, mechanically identical buildings stand at uniform spatial intervals on a linear elastic half-space and they are subjected to anti-plane horizontal impact (Fig. 6b). Note that if, like in many other popular research works, buildings stand on top of a rigid body (shaking table in laboratory experiments), the vibration and the damage given to every identical building will become the same (Fig. 6c). Even when the model building(s) is (are) set on a deformable medium and wave-structure interaction is taken into account, the mechanical behavior of a single building will be different from the collective motion of multiple buildings. Indeed, it has been found that the buildings on a linear elastic half-space significantly interact with each other through the dynamic coupling effect and the resonant (eigen) frequencies of the collective multiple-building system (i.e., town) become lower than that of a single building with the same structural components. Moreover, buildings in a town may really vibrate in a different way (Fig. 6d). The shift of eigenfrequencies and change of structural vibration modes may be called the "town effect" or "city effect". ${ }^{95)}$ Figure 6d depicts semi-analytically obtained distributions of the normalized (absolute values of) displacement (or velocity, acceleration) amplitudes of the foundations and top masses for three different vibration modes of a typical European town consisting of seven mechanically identical buildings. While the fundamental vibration mode of the town gives the building in the middle (number 4) the largest horizontal vibration (and most likely a severer damage level to this building) (left), the second mode (middle) imparts no dynamic horizontal impact to the same building 4 , and the buildings 2 and 6 are under the strongest vibration. In the fourth mode (right), every second building (number 1, 3, 5, 7) experiences much larger vibration than the buildings with even numbers. The eigenfrequency for this fourth mode in the Gemona region is evaluated to be about $3.8 \mathrm{~Hz}$ and it compares well with one of the dominant frequencies ${ }^{96}$ ) for the spectral response estimated from the Friuli 19761977 earthquake sequence at the Tolmezzo-Ambiesta dam (TLM1) accelerograph site. The Friuli case may be one of the actual instances of the seismic "town effect" or "city effect," which may concentrate structural damage to specific structures in a town. It 
a

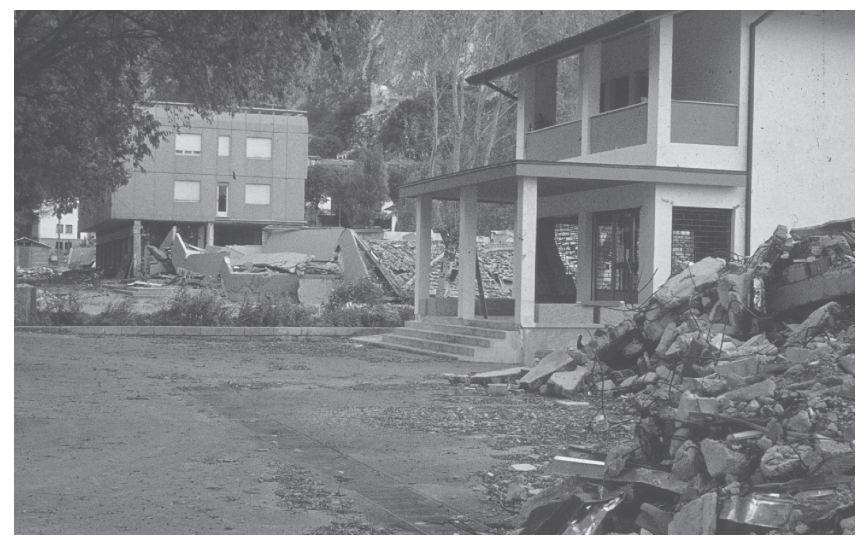

b

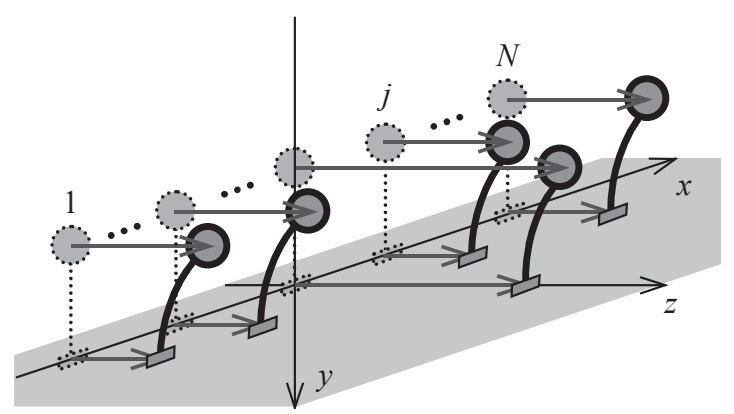

C

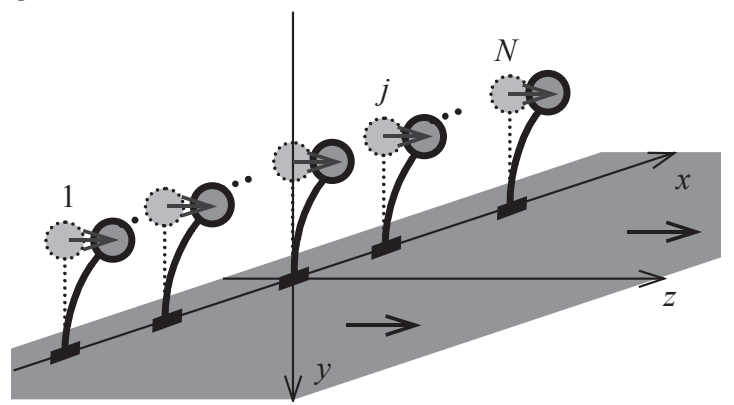

d

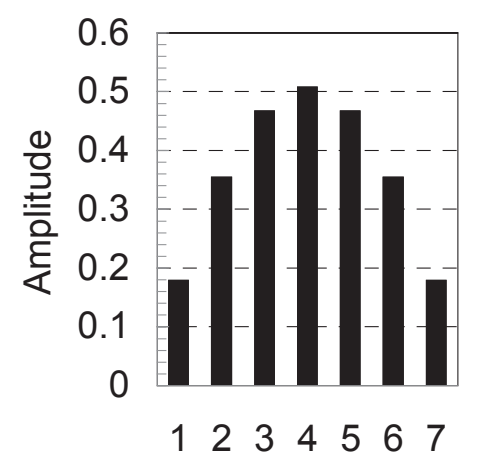

Building number

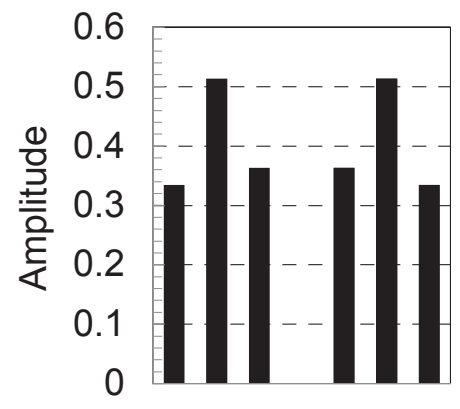

1234567

Building number

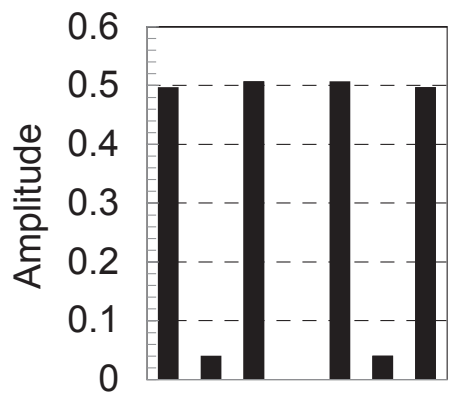

1234567

Building number

Fig. 6. An "unexpected" structural failure pattern in the Gemona region found after the 1976 Friuli, Italy, earthquake. Each adjacent similar surface structure (building) has shown completely alternate structural performance (Photograph courtesy of Professor H. P. Rossmanith at Vienna University of Technology). (b) In the "town model" $N$ identical buildings represented by a top mass, elastic spring and rigid foundation are spatially uniformly set at the horizontal flat surface of a linear elastic half-space. If the dynamic interaction between (vibrations of) the buildings and anti-plane waves in the ground, i.e., wave radiation from the buildings, are taken into account, every building can vibrate totally differently even for a single frequency. (c) The structural failure pattern (a) may not be systematically explained if buildings are conventionally placed on a rigid body (shaking table), which imparts the same vibration (and damage) to each identical building. (d) The normalized (absolute values of) displacement amplitudes of the foundations (and top masses) for the (left) fundamental, (middle) second, and (right) fourth vibration modes of a town of seven mechanically identical buildings. ${ }^{95,97)}$ 
is noteworthy that a similar structural failure pattern was observed in Dinar in Turkey on the occasion of the October 1995 earthquake $\left(M_{w}=6.2\right)$ and the concept of "town effect" may be applied to failure analyses of structures subjected to anti-plane horizontal impact in general, e.g., dynamic collective performance of skyscrapers on the surface or dual tunnels in the underground. ${ }^{95), 97)}$

\section{Dip-slip faulting: seismic rupture moving from surface back into depth}

The final topic in this review handles again the problem of dynamic fault rupture, but that of dip-slip faulting in which the rupture propagates rather vertically, initially from depth. As stated repeatedly, at present it is widespread to inversely evaluate the distributions and histories of fault slips and their influence kinematically from seismograms for both strike-slip and dip-slip earthquakes, but from a dynamics point of view, it is more difficult to treat shallow dip-slip fault rupture than strike-slip one because of the smaller amount of the near-field seismological records of this dip-slip faulting type and also owing to the analytical intricacy in obtaining the mechanical characteristics, especially near the tip of surfacing fault rupture. ${ }^{98)}$ One substantial feature in shallow dip-slip faulting is the asymmetric seismic motion in the proximity of the rupturing fault. Generally, the seismic motion on the hanging wall is much stronger than that on the footwall, and for example, the 1971 San Fernando $\left(M_{w}=6.6\right)$ and the 1994 Northridge $\left(M_{w}=6.7\right)$ earthquakes in California gave systematically more serious structural damage and larger seismic motion on the hanging wall. ${ }^{99}$ In addition to the 1976 Friuli event, ${ }^{100)}$ the 1999 Chi-Chi, Taiwan $\left(M_{w}=\right.$ $7.6),{ }^{101), 102)}$ and the 2008 Iwate-Miyagi Inland, Japan $\left(M_{w}=6.9\right),{ }^{103)}$ earthquakes as well as the more recent one in Nagano has reinforced this standpoint. In the case of the 2014 Nagano-ken Hokubu earthquake $\left(M_{w}=6.3\right)$, the hanging wall on the east side of the Kamishiro fault plane moved upwards with respect to the footwall on the west side, and the kinematic inversion of the main shock and the distribution of the aftershock foci suggest that ruptured fault plane dips steeply, almost vertically near the hypocenter of the main shock (see the cross-sectional view in Fig. 7a). If the cross-section looks geometrically symmetric about the nearly vertical fault plane, the seismic motion on the hanging wall and on the footwall should be more or less of the same magnitude. However, the seismograms deployed by the NIED KiK-net indicate that the peak ground acceleration (PGA) at the Shinshuushin (NGNH27) station on the hanging wall (epicentral distance $19 \mathrm{~km}$ ) is $1.858 \mathrm{~m} / \mathrm{s}^{2}$ while PGA at the Ohmachi-C (NGNH34) station on the footwall with the nearly same epicentral distance $(20 \mathrm{~km})$ is $0.744 \mathrm{~m} / \mathrm{s}^{2}$. Although the station NGNH34 on the footwall looks closer to the surface trace of the fault system, PGA on the hanging wall is about 2.5 times as large as that on the footwall. ${ }^{104)}$

The observed asymmetric seismic motion has been attributed to the strong disturbance in the neighborhood of the tip of dynamic rupture (rupture front wave), ${ }^{98)}$ the trapped wave in the hanging wall, ${ }^{99)}$ or the nonequal mass distributions on the footwall and the hanging wall. ${ }^{99), 102), 105), 106)}$ But, from a rupture and wave dynamics point of view, it has been shown experimentally that, when the primary dip-slip fault rupture, initiated at some depth and propagating upwards in a monolithic linear elastic medium, approaches the free surface, four Rayleightype waves may be generated (Fig. 7b): Two of them run along the free surface as Rayleigh surface waves (R) into the opposite directions to the far field, and the other two move along the ruptured fault plane as interface slip waves (I; on the left and right sides of the interface) back downwards into depth (left column). For an inclined fault plane, in the hanging wall the interface and Rayleigh waves may interact with each other and a shear wave carrying concentrated energy (corner wave $(\mathrm{C})$ ) can be induced to cause stronger disturbances (right). The previously unidentified but numerically predicted ${ }^{107)}$ downward interface and corner waves have been observed through the dynamic photoelastic experiments in Fig. 7b. The corner waves, induced by primary dipslip fault rupture, may be present only in the case of an inclined fault plane, i.e., only when the geometry of the cross-section is asymmetric. If, besides the primary fault rupture, secondary rupture is allowed at a layer interface in a stratified medium, the seismic motion on the hanging wall may become larger than that on the footwall even in a geometrically symmetric case (like the 2014 event in Nagano). This is because (anti-)symmetric rupture pattern can be simply broken due to, for instance, interaction of the waves around the primary rupture front with layer interfaces in such a case. ${ }^{104)}$

In the study of seismic sources, not only the corner waves but also the Rayleigh-type waves traveling along the ruptured fault plane back downwards into depth are not clearly recognized, partially 
a

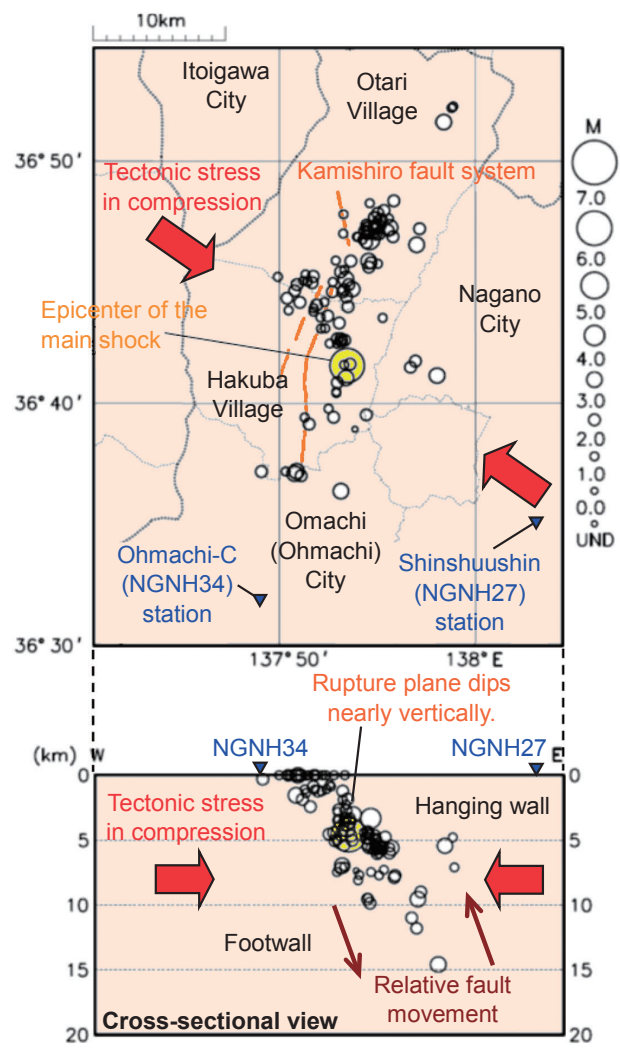

b

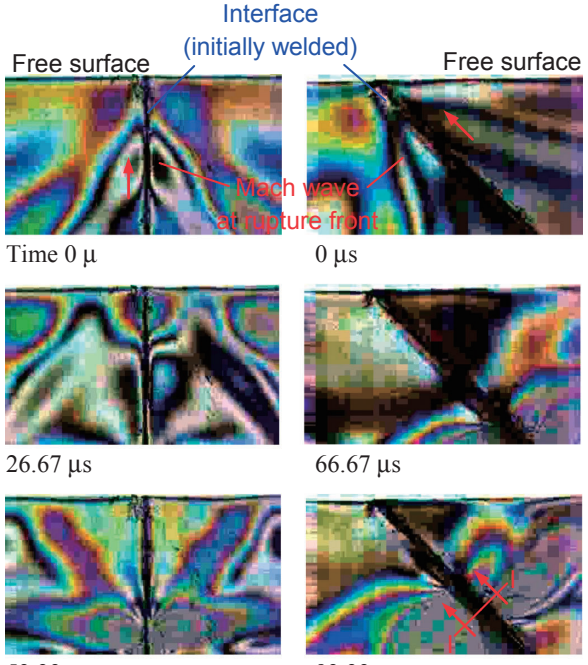

$53.33 \mu \mathrm{s}$

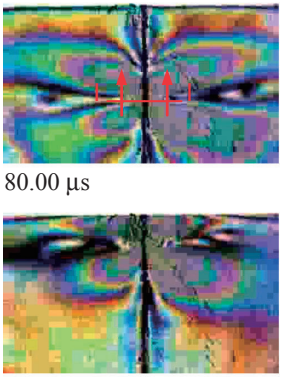

$106.67 \mu \mathrm{s}$

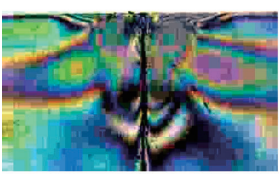

$133.33 \mu \mathrm{s}$

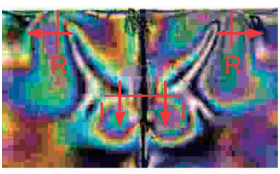

$160.00 \mu \mathrm{s}$

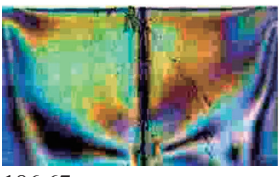

$186.67 \mu \mathrm{s}$
$93.33 \mu \mathrm{s}$

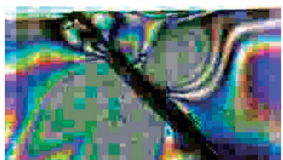

$120.00 \mu \mathrm{s}$

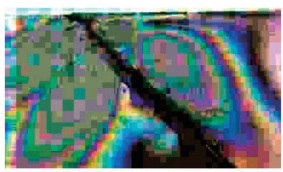

$146.67 \mu \mathrm{s}$

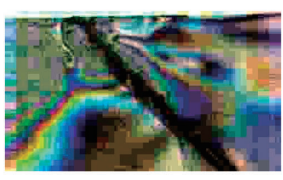

$173.33 \mu \mathrm{s}$

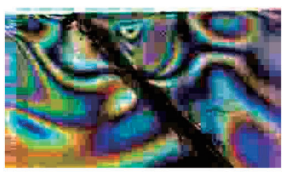

$200.00 \mu \mathrm{s}$

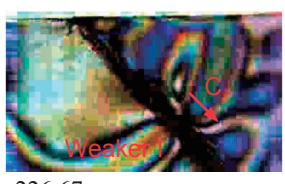

Fig. 7. (a) Distribution of the epicenters of the main- and aftershocks of the 2014 Nagano-ken Hokubu (Kamishiro fault), Japan, earthquake. (b) Photographs experimentally taken utilizing dynamic photoelasticity in conjunction with high-speed cinematography show the time-dependent isochromatic fringe patterns (distributions of $\tau_{\max }$ ) generated near a free surface by supershear dip-slip rupture $\left(V \approx 1.73-1.98 V_{S}\right)$ on a model fault plane (initially welded interface) between two identical transparent, birefringent linear elastic polycarbonate plates. The dip angle is (left) $90^{\circ}$ and (right) $45^{\circ}$, respectively. At an initial stage, a Mach wave may be generated around the upper tip of the supershear rupture. Afterward, strong Rayleigh-type interface waves, involving possible slips (I) and separated from the Mach waves, move upwards along the ruptured interface. Upon approaching the horizontal free surface, the incident interface waves are basically divided into two Rayleigh surface waves (R) and two downward moving interface waves (left). In the inclined fault case (right), the Rayleigh and the downward interface waves are connected to form a corner wave (C) in the hanging wall, which seems much stronger than the downward interface wave in the footwall. ${ }^{104)}$ 
because these waves cannot exist in a standard earthquake model with a fault segment of finite length rupturing only at depth and no receiver is located at depth to detect such downward waves. However, the generation of the downward Rayleightype (interface) waves has been already depicted in the finite difference numerical simulations of borehole blasting in linear elastic solids. In these computations of bottom-to-top blasting, the explosive charge is detonated at the bottom and a detonation front runs along an explosive column upwards to the free surface. Rayleigh waves, created upon detonation, run initially upwards and subsequently downwards along the column. ${ }^{108), 109)}$ Also, it is noteworthy to mention that similar rupture propagation (downward rupture after initial upward one) has been reported, e.g., for the March 2011 earthquake in Japan. ${ }^{110)}$

\section{Conclusions}

This review summarizes some earthquakerelated phenomena that cannot be explained in a systematic way based on conventional kinematic, low-frequency engineering approaches. Evaluations of dominant types and frequencies of the associated seismic waves from the "unfamiliar" structural failure patterns suggest the important role of higherfrequency waves in generating these failures. The "unusual" or "unexpected" findings include the universal critical size for earthquake nucleation, "extraordinary" fast rupture propagation (in the sense of classical fracture mechanics), vertically oscillating higher-frequency waves, open cracks along the crest of a slope, collective behavior of a group of structures (town effect), and the seismic rupture moving back from surface down into depth. Latest experimental study on controlled dynamic fracture in brittle solid materials is revealing that dynamic rupture and wave development in three-dimensional configurations is totally different not only from one-dimensional models but also from two-dimensional ones. ${ }^{2), 111)}$ Further detailed investigations of real three-dimensional dynamics of rupture and waves will enable us to more accurately evaluate and mitigate unexperienced or unexpected seismic hazards.

\section{Acknowledgements}

Valuable comments given by two anonymous reviewers are kindly acknowledged. This work has been financially supported by the Japan Society for the Promotion of Science (JSPS) through the "KAKENHI: Grant-in-Aid for Scientific Research (C)" Program (No. 16K06487).

\section{References}

1) Uenishi, K. (2010) Book review - Seismic Wave Propagation and Scattering in the Heterogeneous Earth, by Haruo Sato and Michael C. Fehler, second print, Modern Acoustics and Signal Processing Series, Springer, 2009. Pure Appl. Geophys. 167, 1583-1584.

2) Uenishi, K., Shigeno, N., Sakaguchi, S., Yamachi, H. and Nakamori, J. (2016) Controlled disintegration of reinforced concrete blocks based on wave and fracture dynamics. Procedia Structural Integrity 2, 350-357.

3) Aki, K. and Richards, P.G. (1980) Quantitative Seismology. W. H. Freeman \& Co., San Francisco.

4) Aki, K. (2005) Eternity of Lamb's problem. J. Seismol. Soc. Jpn., 2nd Ser. 57, 317-319.

5) The Seismological Society of Japan (ed.) (2009) Seismology in Japan: present state and seeds for the 21st century. Special issue of J. Seismol. Soc. Jpn., 2nd Ser., 61, The Seismological Society of Japan, Tokyo.

6) Sato, H. and Fehler, M.C. (1998) Seismic Wave Propagation and Scattering in the Heterogeneous Earth. Springer-Verlag, New York.

7) Itoh, H., Okada, T., Funato, A., Uenishi, K., Uchimura, T., Uno, H., Ookuma, N., Kawasaki, S., Suzuki, K., Nakajima, M., Hayano, K., Yamada, S. and Yoshida, J. (2014) JGS 25612012: method for multi-stage cyclic undrained triaxial compression test on rocks, and JGS 2562-2012: method for cyclic undrained triaxial compression test to determine fatigue properties of rocks. In New Japanese Geotechnical Standards (Fiscal Year 2013). The Japanese Geotechnical Society (JGS), Tokyo, pp. 35-103.

8) Kanamori, H. (2004) The diversity of the physics of earthquakes. Proc. Jpn. Acad., Ser. B 80, 297316.

9) Ide, S. (2014) Modeling fast and slow earthquakes at various scales. Proc. Jpn. Acad., Ser. B 90, 259277.

10) Uenishi, K., Rossmanith, H.P. and Scheidegger, A.E. (1999) Rayleigh pulse - dynamic triggering of fault slip. Bull. Seismol. Soc. Am. 89, 12961312.

11) Scheidegger, A.E. (1982) Principles of Geodynamics (Third, Completely Revised Edition). SpringerVerlag, Berlin.

12) Ruina, A. (1983) Slip instability and state variable friction laws. J. Geophys. Res. 88, 10,359-10,370.

13) Tse, S.T. and Rice, J.R. (1986) Crustal earthquake instability in relation to the depth variation of frictional slip properties. J. Geophys. Res. 91, 9452-9472.

14) Okubo, P.G. (1989) Dynamic rupture modeling with laboratory-derived constitutive relations. J. Geophys. Res. 94, 12,321-12,335.

15) Dieterich, J.H. (1992) Earthquake nucleation on faults with rate- and state-dependent strength. Tectonophysics 211, 115-134.

16) Rice, J.R. and Ben-Zion, Y. (1996) Slip complexity 
in earthquake fault models. Proc. Natl. Acad. Sci. U.S.A. 93, 3811-3818.

17) Ohnaka, M. (2000) A physical scaling relation between the size of an earthquake and its nucleation zone size. Pure Appl. Geophys. 157, $2259-2282$.

18) Campillo, M. and Ionescu, I.R. (1997) Initiation of antiplane shear instability under slip dependent friction. J. Geophys. Res. 102, 20,363-20,371.

19) Ionescu, I.R. and Campillo, M. (1999) Influence of the shape of friction law and fault finiteness on the duration of initiation. J. Geophys. Res. 104, 3013-3024.

20) Favreau, P., Campillo, M. and Ionescu, I.R. (1999) Initiation of in-plane shear instability under slipdependent friction. Bull. Seismol. Soc. Am. 89, $1280-1295$.

21) Dascalu, C., Ionescu, I.R. and Campillo, M. (2000) Fault finiteness and initiation of dynamic shear instability. Earth Planet. Sci. Lett. 177, 163-176.

22) Lapusta, N., Rice, J.R., Ben-Zion, Y. and Zheng, G. (2000) Elastodynamic analysis for slow tectonic loading with spontaneous rupture episodes on faults with rate- and state-dependent friction. J. Geophys. Res. 105, 23,765-23,789.

23) Lapusta, N. and Rice, J.R. (2003) Nucleation and early seismic propagation of small and large events in a crustal earthquake model. J. Geophys. Res. 108, ESE8. doi: 10.1029/2001JB000793.

24) Uenishi, K. and Rice, J.R. (2003) Universal nucleation length for slip-weakening rupture instability under nonuniform fault loading. J. Geophys. Res. 108, ESE17. doi: 10.1029/2001JB001681.

25) Uenishi, K. and Rice, J.R. (2004) Three-dimensional rupture instability of a slip-weakening fault under heterogeneous loading. Eos Trans. AGU 85, Fall Meet. Suppl., Abstract S13E-04.

26) Rummel, F., Alheid, H.J. and Frohn, C. (1978) Dilatancy and fracture-induced velocity changes in rock and their relation to frictional sliding. Pure Appl. Geophys. 116, 743-764.

27) Rice, J.R. (1980) The mechanics of earthquake rupture. In Physics of the Earth's Interior (eds. Dziewonski, A.M. and Boschi, E.). Italian Physical Society and North-Holland Publ. Co., Amsterdam, pp. 555-649.

28) Wong, T.-F. (1986) On the normal stress dependence of the shear fracture energy. In Earthquake Source Mechanics (eds. Das, S., Boatwright, J. and Scholz, C.H.). American Geophysical Union, Washington, D.C., pp. 1-11.

29) Harris, R.A., Archuleta, R.J. and Day, S.M. (1991) Fault steps and the dynamic rupture process: 2-D numerical simulations of a spontaneously propagating shear fracture. Geophys. Res. Lett. 18, $893-896$

30) Ide, S. and Takeo, M. (1997) Determination of constitutive relations of fault slip based on seismic wave analysis. J. Geophys. Res. 102, 27,37927,391 .

31) Olsen, K.B., Madariaga, R. and Archuleta, R.J. (1997) Three-dimensional dynamic simulation of the 1992 Landers earthquake. Science 278, 834838 .

32) Colombelli, S., Zollo, A., Festa, G. and Picozzi, M. (2014) Evidence for a difference in rupture initiation between small and large earthquakes. Nat. Commun. 5, 3958. doi: 10.1038/ncomms4958.

33) Shaw, B.E. and Rice, J.R. (2000) Existence of continuum complexity in the elastodynamics of repeated fault ruptures. J. Geophys. Res. 105, $23,791-23,810$

34) Guatteri, M. and Spudich, P. (2000) What can strong motion data tell us about slip-weakening fault friction laws? Bull. Seismol. Soc. Am. 90, 98-116.

35) Spudich, P. and Cranswick, E. (1984) Direct observation of rupture propagation during the 1979 Imperial Valley earthquake using a short beseline accelerometer array. Bull. Seismol. Soc. Am. 74, 2083-2114.

36) Bouchon, M., Toksöz, M.N., Karabulut, H., Bouin, M.-P., Dietrich, M., Aktar, M. and Edie, M. (2002) Space and time evolution of rupture and faulting during the 1999 Izmit (Turkey) earthquake. Bull. Seismol. Soc. Am. 92, 256-266.

37) Bouchon, M. and Vallée, M. (2003) Observation of long supershear rupture during the magnitude 8.1 Kunlunshan earthquake. Science 301, 824-826.

38) Oglesby, D.D., Dreger, D.S., Harris, R.A., Ratchkovski, N. and Hansen, R. (2004) Inverse kinematic and forward dynamic models of the 2002 Denali Fault earthquake, Alaska. Bull. Seismol. Soc. Am. 94, S214-S233.

39) Ravi-Chandar, K. and Knauss, W.G. (1984) An experimental investigation into dynamic fracture. I. crack initiation and crack arrest. Int. J. Fract. 25, 247-262.

40) Ravi-Chandar, K. and Knauss, W.G. (1984) An experimental investigation into dynamic fracture. II. microstructural aspects. Int. J. Fract. 26, 6580.

41) Ravi-Chandar, K. and Knauss, W.G. (1984) An experimental investigation into dynamic fracture. III. steady state crack propagation and crack branching. Int. J. Fract. 26, 141-154.

42) Ravi-Chandar, K. and Knauss, W.G. (1984) An experimental investigation into dynamic fracture. IV. on the interaction of stress waves with propagating cracks. Int. J. Fract. 26, 189-200.

43) Sharon, E. and Fineberg, J. (1996) Microbranching instability and the dynamic fracture of brittle materials. Phys. Rev. B 54, 7128-7139.

44) Rice, J.R. (2001) New perspectives on crack and fault dynamics. In Mechanics for a New Millennium (eds. Aref, H. and Phillips, J.W.). Kluwer Academic Publishers, Dordrecht, pp. 1-23.

45) Uenishi, K. and Rossmanith, H.P. (2002) Stability of dynamically propagating cracks in brittle materials. Acta Mech. 156, 179-192.

46) Willis, J.R. (1973) Self-similar problems in elastodynamics. Philos. Trans. R. Soc. Lond. 274, 435491.

47) Atkinson, C. (1977) Dynamic crack problems in 
dissimilar media. In Mechanics of Fracture Vol. 4 (ed. Sih, G.C.). Noordhoff, Leyden, pp. 213-248.

48) Weertman, J. (1980) Unstable slippage across a fault that separates elastic media of different elastic constants. J. Geophys. Res. 85, 1455-1461.

49) Andrews, D.J. and Ben-Zion, Y. (1997) Wrinkle-like slip pulse on a fault between different materials. J. Geophys. Res. 102, 553-571.

50) Day, S.M. (1991) Numerical simulation of fault propagation with interface separation. Eos Trans. AGU 72, Fall Meet. Suppl., 486.

51) Mora, P. and Place, D. (1994) Simulation of the frictional stick-slip instability. Pure Appl. Geophys. 143, 61-87.

52) Rosakis, A.J., Xia, K., Lykotrafitis, G. and Kanamori, H. (2007) Dynamic shear rupture in frictional interfaces: speeds, directionality, and modes. In Treatise on Geophysics (Second Edition) Vol. 4. Elsevier, Amsterdam, pp. 183213.

53) Heaton, T.H. (1990) Evidence for and implications of self-healing pulses of slip in earthquake rupture. Phys. Earth Planet. Inter. 64, 1-20.

54) Wald, D.J. (1996) Slip history of the 1995 Kobe, Japan, earthquake determined from strong motion, teleseismic, and geodetic data. J. Phys. Earth 44, 489-503.

55) Pitarka, A., Irikura, K., Iwata, T. and Sekiguchi, H. (1998) Three-dimensional simulation of the nearfault ground motion for the 1995 Hyogo-ken Nanbu (Kobe), Japan, earthquake. Bull. Seismol. Soc. Am. 88, 428-440.

56) Buehler, M.J. and Gao, H. (2006) Dynamical fracture instabilities due to local hyperelasticity at crack tips. Nature 439, 307-310.

57) Zhou, X., Hac, Q., Zhanga, Y. and Zhua, K. (2004) Analysis of deformation localization and the complete stress-strain relation for brittle rock subjected to dynamic compressive loads. Int. J. Rock Mech. Min. Sci. 41, 311-319.

58) Uenishi, K. (2012) Experimental study on the supersonic crack propagation in a hyperelastic rubber material. Trans. Jpn. Soc. Mech. Eng. A 78, 628-631.

59) Uenishi, K. (2012) Elastodynamic analysis of underground structural failures induced by seismic body waves. ASME J. Appl. Mech. 79, 031014-1031014-10

60) Uenishi, K. and Sakurai, S. (2008) Recent developments in rock mechanics as applied to earthquake dynamics. Geomech. Tunnel. 1, 368-373.

61) Konagai, K., Johansson, J., Zafeirakos, A., Numada, M. and Sadr, A. (2005) Damage to Tunnels in the October 23, 2004 Chuetsu earthquake. JSCE J. Earthq. Eng. 28, 75-1-75-8.

62) Pao, Y.-H. (1962) Dynamical stress concentration in an elastic plate. ASME J. Appl. Mech. 29, 299305.

63) Mow, C.C. and McCabe, W.L. (1963) Dynamic stresses in an elastic cylinder. J. Eng. Mech. Div. 89, 21-41.

64) Cheng, S.L. (1969) Multiple scattering of elastic waves by parallel cylinders. ASME J. Appl. Mech. 36, 523-527.

65) Mow, C.C. and Mente, L.J. (1963) Dynamic stresses and displacements around cylindrical discontinuities due to plane harmonic shear waves. ASME J. Appl. Mech. 30, 598-604.

66) Lee, V.W. and Trifunac, M.D. (1979) Response of tunnels to incident SH-waves. J. Eng. Mech. Div. 105, 643-659.

67) Balendra, T., Thambiratnam, D.P., Koh, C.G. and Lee, S.L. (1984) Dynamic response of twin circular tunnels due to incident SH-waves. Earthquake Eng. Struct. Dynam. 12, 181-201.

68) Uenishi, K. and Sakurai, S. (2000) Characteristic of the vertical seismic waves associated with the 1995 Hyogo-ken Nanbu (Kobe), Japan earthquake estimated from the failure of the Daikai underground station. Earthquake Eng. Struct. Dynam. 29, 813-821.

69) Bungum, H. and Olesen, O. (2004) The 31st of August 1819 Luroy earthquake revisited. Norwegian J. Geol. 85, 245-252.

70) Birch, F.S. (1966) An earthquake recorded at sea. Bull. Seismol. Soc. Am. 56, 361-366.

71) Mogi, K. and Mochizuki, H. (1980) Observation of high frequency seismic waves by a hydrophone just above the focal region of the 1980 Izu-Hantooki earthquake and a precise estimate of location of the submarine earthquake fault. Bull. Earthq. Res. Inst. 55, 1017-1041.

72) Ambraseys, N. (1985) Short communication: a damaging seaquake. Earthquake Eng. Struct. Dynam. 13, 421-424.

73) Kawata, Y. (1996) Seaquakes felt on board a ship, seismic waves seen in a tunnel. Kagaku 66, 70-71 (in Japanese).

74) O'Loughlin, K.F. and Lander, J.F. (2003) Caribbean Tsunamis. Springer Science + Business Media, Dordrecht.

75) Maehara, H. and Sakurai, S. (2012) On representative examples of instantaneous structural failures due to earthquakes. Proc. 2012 Kinki Regional Development Bureau Res. Symp., 6 pages (in Japanese).

76) Baba, E. (1987) A study on the effect of seaquakes on a floating body. J. Soc. Naval Arch. Jpn. 162, 90-98.

77) Kiyokawa, T. and Inada, Y. (1989) Mechanism of occurrence of seaquakes. Ann. J. Coast. Eng. JSCE 36, 734-738 (in Japanese).

78) Mochizuki, K., Kihara, H., Takagi, K. and Naito, S. (1997) Effects of seaquake on coastal floating structure. J. Kansai Soc. Naval Arch. Jpn. 227, $127-136$.

79) Jang, R.-D. and Higo, Y. (2001) Free surface wave elevation interacted with compression waves in seawater caused by earthquake. J. Soc. Naval Arch. Jpn. 189, 81-85.

80) Uenishi, K. and Sakurai, S. (2014) The generation of seaquakes and its impact on floating bodies. Int. J. Prot. Struct. 5, 207-218.

81) Uenishi, K. and Sakurai, S. (2015) Dynamic tensile 
cracking in slopes possibly induced by Rayleigh surface waves. Geomech. Geoeng. 10, 212-222.

82) Sitar, N., Clough, G.W. and Bachus, R.C. (1980) Behavior of Weakly Cemented Soil Slopes under Static and Seismic Loading. Stanford University, Stanford.

83) Ashford, S.A. and Sitar, N. (1997) Analysis of topographic amplification of inclined shear waves in a steep coastal bluff. Bull. Seismol. Soc. Am. 87, 692-700.

84) Kaiser, A., Holden, C., Beavan, J., Beetham, D., Benites, R., Celentano, A., Collett, D., Cousins, J., Cubrinovski, M., Dellow, G., Denys, P., Fielding, E., Fry, B., Gerstenberger, M., Langridge, R., Massey, C., Motagh, M., Pondard, N., McVerry, G., Ristau, J., Stirling, M., Thomas, J., Uma, S.R. and Zhao, J. (2012) The Mw 6.2 Christchurch earthquake of February 2011: preliminary report. N.Z. J. Geol. Geophys. $\mathbf{5 5}, 67-90$.

85) Ashford, S.A., Sitar, N., Lysmer, J. and Deng, N. (1997) Topographic effects on the seismic response of steep slopes. Bull. Seismol. Soc. Am. 87, 701709 .

86) Uenishi, K. (2010) On a possible role of Rayleigh surface waves in dynamic slope failures. Int. J. Geomech. 10, 153-160.

87) Furumura, T. and Kennett, B.L.N. (2005) Subduction zone guided waves and the heterogeneity structure of the subducted plate: intensity anomalies in northern Japan. J. Geophys. Res. 110, B10302. doi: 10.1029/2004JB003486.

88) Wirgin, A. and Bard, P.-Y. (1996) Effects of buildings on the duration and amplitude of ground motion in Mexico City. Bull. Seismol. Soc. Am. 86, 914-920.

89) Clouteau, D. and Aubry, D. (2001) Modification of ground motion in dense urban areas. J. Comput. Acoust. 6, 1659-1675.

90) Guéguen, P., Bard, P.-Y. and Chavez-Garcia, F.J. (2002) Site-city seismic interaction in Mexico City like environment: an analytic study. Bull. Seismol. Soc. Am. 92, 794-811.

91) Tsogka, C. and Wirgin, A. (2003) Simulation of seismic response in an idealized city. Soil. Dyn. Earthquake Eng. 23, 391-402.

92) Boutin, C. and Roussillon, P. (2004) Assessment of the urbanization effect on seismic response. Bull. Seismol. Soc. Am. 94, 251-268.

93) Kham, M., Semblat, J.-F., Bard, P.-Y. and Dangla, P. (2006) Seismic site-city interaction: main governing phenomena through simplified numerical models. Bull. Seismol. Soc. Am. 96, 19341951.

94) Ghergu, M. and Ionescu, I.R. (2009) Structure-soilstructure coupling in seismic excitation and "city effect". Int. J. Eng. Sci. 47, 342-354.

95) Uenishi, K. (2010) The town effect: dynamic interaction between a group of structures and waves in the ground. Rock Mech. Rock Eng. 43, 811-819.
96) Barnaba, C., Priolo, E., Vuan, A. and Romanelli, M. (2007) Site effect of the strong-motion site at Tolmezzo-Ambiesta Dam in northeastern Italy. Bull. Seismol. Soc. Am. 97, 339-346.

97) Uenishi, K. (2013) "Unexpected" failure patterns and dynamic collective behaviour of an assembly of buildings subjected to horizontal impact. Eng. Fail. Anal. 35, 125-132.

98) Madariaga, R. (2003) Radiation from a finite reverse fault in a half space. Pure Appl. Geophys. 160, $555-577$.

99) Oglesby, D.D., Archuleta, R.J. and Nielsen, S.B. (1998) Earthquakes on dipping faults; the effects of broken symmetry. Science 280, 1055-1059.

100) Zollo, A., Bobbio, A., Emolo, A., Herrero, A. and De Natale, G. (1997) Modelling of ground acceleration in the near source range: the case of 1976, Friuli earthquake $(\mathrm{M}=6.5)$, northern Italy. J. Seismol. 1, 305-319.

101) Kao, H. and Chen, W.P. (2000) The Chi-Chi earthquake sequence: active, out-of-sequence thrust faulting in Taiwan. Science 288, 23462349.

102) Oglesby, D.D. and Day, S.M. (2001) Fault geometry and the dynamics of the 1999 Chi-Chi (Taiwan) earthquake. Bull. Seismol. Soc. Am. 91, 10991111.

103) Aoi, S., Kunugi, T. and Fujiwara, H. (2008) Trampoline effect in extreme ground motion. Science 322, 727-730.

104) Uenishi, K. (2015) Dynamic dip-slip fault rupture in a layered geological medium: broken symmetry of seismic motion. Eng. Fail. Anal. 58, 380-393.

105) Davis, P.M. and Knopoff, L. (1991) The dipping antiplane crack. Geophys. J. Int. 106, 581-585.

106) Oglesby, D.D., Archuleta, R.J. and Nielsen, S.B. (2000) Dynamics of dip-slip faulting; explorations in two dimensions. J. Geophys. Res. 105, 13,64313,653 .

107) Uenishi, K. and Madariaga, R.I. (2005) Surface breaking dip-slip fault: its dynamics and generation of corner waves. Eos Trans. AGU 86, Fall Meet. Suppl., Abstract S34A-03.

108) Rossmanith, H.P., Uenishi, K. and Kouzniak, N. (1997) Blast wave propagation in rock mass part I: monolithic medium. Fragblast 1, 317-359.

109) Uenishi, K. and Rossmanith, H.P. (1998) Blast wave propagation in rock mass - part II: layered media. Fragblast 2, 39-77.

110) Ide, S., Baltay, A. and Beroza, G.C. (2011) Shallow dynamic overshoot and energetic deep rupture in the $2011 \mathrm{Mw} 9.0$ Tohoku-oki earthquake. Science 332, 1426-1429.

111) Uenishi, K., Yamachi, H., Yamagami, K. and Sakamoto, R. (2014) Dynamic fragmentation of concrete using electric discharge impulses. Construct. Build. Mater. 67, 170-179. 


\section{Profile}

Koji Uenishi was born in Hyogo Prefecture in 1970. After receiving Master of Engineering degree from the University of Tokyo in 1995, he initiated his research career as a research associate at the Institute of Mechanics, Vienna University of Technology in Austria where he earned Doctor of Science degree in 1997. He then moved to Kobe University in 1998 as an assistant professor and was promoted to an associate professor of the Research Center for Urban Safety and Security in 2006. In the meanwhile, from 2000 to 2002, he stayed as a visiting scientist at the Division of Engineering and Applied Sciences and Department of Earth and Planetary Sciences of Harvard University in the United States of America. He returned to the School of Engineering of the University of Tokyo in 2012. By employing an engineering approach of applied and experimental

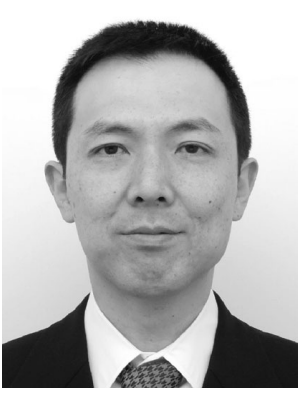
mechanics, he is studying fracture (rupture) dynamics of solids, in particular, that of the solid Earth and physics of earthquakes. He is also developing practical technologies for precisely controlled fracture in solid materials. He was awarded, among others, the Leopold Mueller Award of the Austrian Society for Geomechanics in 1999, the Young Scientist Award of the Seismological Society of Japan in 2006, the Young Scientists' Prize of the Ministry of Education, Culture, Sports, Science and Technology of Japan in 2009, and the JSPS Prize in 2016. 